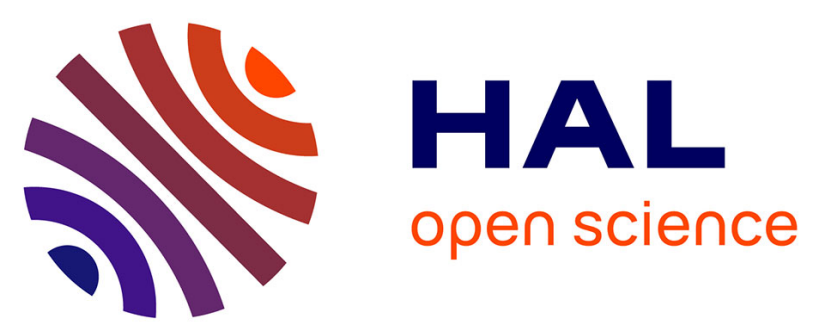

\title{
A RIEMANN SOLUTION APPROXIMATION BASED ON THE ZERO DIFFUSION-DISPERSION LIMIT OF DAFERMOS REFORMULATION TYPE PROBLEM
}

Christophe Berthon, Marianne Bessemoulin-Chatard, Anaïs Crestetto, Françoise Foucher

\section{To cite this version:}

Christophe Berthon, Marianne Bessemoulin-Chatard, Anaïs Crestetto, Françoise Foucher. A RIEMANN SOLUTION APPROXIMATION BASED ON THE ZERO DIFFUSION-DISPERSION LIMIT OF DAFERMOS REFORMULATION TYPE PROBLEM. Calcolo, In press. hal-02192729

\section{HAL Id: hal-02192729 \\ https://hal.science/hal-02192729}

Submitted on 24 Jul 2019

HAL is a multi-disciplinary open access archive for the deposit and dissemination of scientific research documents, whether they are published or not. The documents may come from teaching and research institutions in France or abroad, or from public or private research centers.
L'archive ouverte pluridisciplinaire HAL, est destinée au dépôt et à la diffusion de documents scientifiques de niveau recherche, publiés ou non, émanant des établissements d'enseignement et de recherche français ou étrangers, des laboratoires publics ou privés. 


\title{
A RIEMANN SOLUTION APPROXIMATION BASED ON THE ZERO DIFFUSION-DISPERSION LIMIT OF DAFERMOS REFORMULATION TYPE PROBLEM
}

\author{
CHRISTOPHE BERTHON*, MARIANNE BESSEMOULIN-CHATARD*, ANAÏS \\ CRESTETTO*, AND FRANÇOISE FOUCHER*†
}

AMS subject classifications. 65N06, 35L65, 35L67

Key words. Diffusive-dispersive system, conservation laws, nonclassical shock waves, boundary value problems, finite difference scheme, discrete solution existence

\begin{abstract}
In the present work, a new numerical strategy is designed to approximate the Riemann solutions of systems of conservation laws. Here, the main difficulty comes from the definition of the discontinuous solutions. Indeed, the shock solutions are no longer selected by entropy criterion but they are defined as the zero limit of a diffusive-dispersive system. As a consequence, the solutions of interest may contain non classical shocks. In order to derive a suitable numerical approach, the Dafermos diffusion technique is adopted here. Then, the PDE initial value problem is reformulated as an ODE boundary value problem. A fourth-order finite difference scheme is introduced to approximate the solution of this ODE boundary value problem. In this work, a particular attention is paid on the existence of discrete solutions and several numerical experiments illustrate the relevance of the derived numerical strategy.
\end{abstract}

1. Introduction. The present work aims at deriving a new numerical strategy to approximate the Riemann solutions of a hyperbolic system of conservation laws in the following form:

$$
\partial_{t} \mathbf{u}+\partial_{x} \mathbf{f}(\mathbf{u})=0, \quad x \in \mathbb{R}, t>0 .
$$

The system is completed with an initial data given by

$$
\mathbf{u}(x, 0)= \begin{cases}\mathbf{u}_{L} & \text { if } x<0, \\ \mathbf{u}_{R} & \text { if } x>0,\end{cases}
$$

where $\mathbf{u}_{L}$ and $\mathbf{u}_{R}$ are two given constant states assumed to belong to $\Omega \subset \mathbb{R}^{d}$, an open subset of admissible states, with $d \geq 1$. Here, $\mathbf{u}(x, t) \in \Omega$ stands for the unknown state vector and $\mathbf{f}: \Omega \rightarrow \mathbb{R}^{d}$ is a given smooth flux function.

It is worth noticing that we do not impose some hyperbolicity criterion. More precisely, as soon as $d \geq 2$, the Jacobian matrix $\nabla_{\mathbf{u}} \mathbf{f}$ may admit complex eigenvalues. Despite this stability failure of the system under consideration, it is well-known that such conservation laws may develop, in finite time, discontinuous solutions. In this work, contrary to the usual approach $[6,7,10,12-14]$, we do not enforce entropy inequalities to select, among the weak solutions of (1.1)-(1.2), the physical relevant one. Here, according to $[11,17]$, the solution $\mathbf{u} \in \Omega$ of interest is assumed to be the limit solution, when some small parameter $\varepsilon$ tends to zero, of the following diffusivedispersive system of conservation laws:

$$
\partial_{t} \mathbf{u}^{\varepsilon}+\partial_{x} \mathbf{f}\left(\mathbf{u}^{\varepsilon}\right)=\varepsilon \beta \partial_{x x} \mathbf{D}\left(\mathbf{u}^{\varepsilon}\right)+\varepsilon^{2} \gamma \partial_{x x x} \mathbf{D}\left(\mathbf{u}^{\varepsilon}\right),
$$

\footnotetext{
${ }^{*}$ Laboratoire de Mathématiques Jean Leray, CNRS UMR 6629, Université de Nantes, 2 rue de la Houssinière, BP 92208, 44322 Nantes, France.

†École Centrale de Nantes, 1 rue de la Noë, BP 92101, 44321 Nantes cedex 3, France.
} 
endowed with the initial data (1.2). The given smooth function $\mathbf{D}: \Omega \rightarrow \mathbb{R}^{d}$ may eventually be restricted according to some assumptions (for instance, see [5]).

It is worth noticing that, from the work by LeFloch [14], it is known that the solutions of (1.3) depend on the ratio $\beta / \gamma$. Then, the sought Riemann solution of (1.1)-(1.2), obtained in the limit of $\mathbf{u}^{\varepsilon}$ as $\varepsilon$ tends to zero, also depends on such a ratio.

Now, it is essential to emphasize that the solutions of (1.1)-(1.2) of interest may violate the usual entropy conditions (for instance, see $[5,14,17]$ ). In other words, the expected weak solution may contain nonclassical shock waves. It is thus crucial to underline that the expected Riemann solution is very complex to be reached. In [14] (see also $[17,20,21]$ ), existence results are given for both scalar and system of conservation laws. But, as soon as the flux function is nonconvex, even by considering scalar hyperbolic equations, the exact Riemann solution of (1.1)-(1.2), seen as the limit solution of the diffusive-dispersive system (1.3), generally cannot be exhibited.

From a numerical point of view, it turns out to be very challenging to propose an approximation of $\mathbf{u}=\lim _{\varepsilon \rightarrow 0} \mathbf{u}^{\varepsilon}$. Indeed, the natural fan structure (rarefaction and shock waves) is not only defined by the flux function $\mathbf{f}$, but it also depends on the diffusive and the dispersive coefficients in (1.3). As a consequence, the approximation of the diffusive-dispersive system (1.3) is privileged and the obtained approximate solution must then be considered in the limit of $\varepsilon$ to zero. For all these reasons, the adopted discretization must be able to correctly capture the effects issuing from the right-hand side in (1.3). This means that the derived scheme must be, at least, third-order of accuracy and the space discretization, namely the mesh size, has to be coherent with $\varepsilon \beta \mathbf{D}(\mathbf{u})$ and $\varepsilon^{2} \gamma \mathbf{D}(\mathbf{u})$. This last condition may make the numerical method very costly since both diffusion and dispersion terms are governed by the, eventually very small, quantity $\varepsilon$. A priori, this means that the mesh size must be also governed by $\varepsilon$.

The objective is thus to derive numerical techniques able to correctly capture the weak solutions of (1.3). Several methods have been introduced by LeFloch and coauthors $[2,5,15,17]$ during the fifteen last years. In [16] (see also [5]), a scalar conservation law, with $d=1$, is considered. The authors have introduced a third-order in both space and time finite difference scheme. They exhibit numerical solutions containing nonclassical undercompressive shock waves. More recently, in $[8,15]$, the authors have constructed a well-controlled diffusion scheme to approximate nonclassical shocks.

When focusing on the approximation of the Riemann solutions of (1.1)-(1.2), more specific numerical strategies can be considered. Indeed, it is well known that the Riemann solutions are self-similar and only depend on the variable $\xi=x / t$. From [14] (for instance, see also [3,17,21]), in order to enforce the solution of (1.3) to be self-similar, we adopt a reformulation of the diffusive-dispersive system according to the Dafermos viscosity approach [6] as follows:

$$
\partial_{t} \mathbf{u}^{\varepsilon}+\partial_{x} \mathbf{f}\left(\mathbf{u}^{\varepsilon}\right)=\varepsilon t \beta \partial_{x x} \mathbf{D}\left(\mathbf{u}^{\varepsilon}\right)+\varepsilon^{2} t^{2} \gamma \partial_{x x x} \mathbf{D}\left(\mathbf{u}^{\varepsilon}\right) .
$$

With the change of variables given by

$$
\mathbf{u}^{\varepsilon}(x, t)=\mathbf{u}(\xi) \quad \text { with } \xi=\frac{x}{t},
$$

we are, in fact, considering the following ODE system:

$$
-\xi \mathbf{u}^{\prime}+\mathbf{f}(\mathbf{u})^{\prime}=\varepsilon \beta \mathbf{D}(\mathbf{u})^{\prime \prime}+\varepsilon^{2} \gamma \mathbf{D}(\mathbf{u})^{\prime \prime \prime},
$$


supplemented by the limit boundary conditions defined by

$$
\lim _{\xi \rightarrow-\infty} \mathbf{u}(\xi)=\mathbf{u}_{L} \quad \text { and } \quad \lim _{\xi \rightarrow+\infty} \mathbf{u}(\xi)=\mathbf{u}_{R}
$$

At this stage, from a numerical point of view, we have to deal with boundary conditions stated at infinity. Following ideas introduced in [11], we will adopt boundary conditions given by

$$
\mathbf{u}(-\ell)=\mathbf{u}_{L} \quad \text { and } \quad \mathbf{u}(\ell)=\mathbf{u}_{R}
$$

with $\ell>0$ large enough. As a consequence, we have now to consider the approximation of the solutions of (1.5)-(1.6). Such a numerical strategy has been adopted in [20], where the authors introduced a continuation technique to approximate the solutions in the viscous case, i.e. with $\gamma=0$. They exhibited suitable numerical approximations to justify the relevance of the Dafermos diffusion [6] in designing numerical approaches based on the equivalent ODE problem (1.5). In [20], they used numerical experiments to study the bifurcation diagram, represented by a figure characterizing the structure of the solution according to the considered Riemann problem, but the numerical analysis of the derived scheme was not presented.

The present paper stays in the spirit of [20] by considering the Dafermos reformulation of the viscosity and the capillarity given by system (1.5). Adopting a scalar conservation law and a p-system like model, we introduce new numerical schemes to approximate the solution of the boundary value problem (1.5)-(1.6). The discrete solution is obtained by solving a nonlinear system of equations and this discrete solution is proved to exist. Next, equipped with the suitable scheme, several numerical experiments illustrate the relevance of the derived numerical method.

The paper is organized as follows. In the next section, a scalar conservation law is considered. To approximate the solutions of this model, we introduce a fourth-order finite difference scheme. The discrete solution of the boundary value problem under consideration satisfies a nonlinear equation stated in $\mathbb{R}^{N}$, where $N$ is the number of cells within the mesh. We prove the existence of the discrete solution. In Section 3 , we propose an extension to a p-system like model introduced in [11]. Involving a suitable reformulation of the model, the boundary value problem is reduced to a scalar ODE with a singularity in $\xi=0$. We develop a fourth-order numerical scheme able to correctly deal with the singularity. Once again an existence result of the approximate solution is established. The last section is devoted to numerical experiments. Since a large system of nonlinear equations must be solved to get the expected solutions, we adopt here a continuation damping Newton method. This algorithm is thus applied to our problems. Several numerical results are displayed to illustrate the interest of such an approach. In particular, we show the ability of the designed method in capturing nonclassical shock waves.

2. Scalar conservation laws. In this section, we consider a diffusive-dispersive scalar equation given by

$$
\partial_{t} w^{\varepsilon}+\partial_{x} f\left(w^{\varepsilon}\right)=\varepsilon t \beta \partial_{x x} w^{\varepsilon}+\varepsilon^{2} t^{2} \gamma \partial_{x x x} w^{\varepsilon}, \quad x \in \mathbb{R}, t>0,
$$

where $f: \mathbb{R} \rightarrow \mathbb{R}$ is a given smooth function.

Arguing the change of variables

$$
w^{\varepsilon}(x, t)=w(\xi) \quad \text { with } \xi=\frac{x}{t},
$$


the unknown $w$ is now solution of the following equation:

$$
-\xi w^{\prime}+f(w)^{\prime}=\varepsilon \beta w^{\prime \prime}+\varepsilon^{2} \gamma w^{\prime \prime \prime} .
$$

According to (1.6), this equation is endowed with boundary conditions given by

$$
w(-\ell)=w_{L} \quad \text { and } \quad w(\ell)=w_{R},
$$

with $\ell>0$ large enough.

Now, to approximate the solutions of (2.1)-(2.2), we suggest a discretization of the interval $[-\ell, \ell]$. We consider a uniform mesh made of $N+1$ cells $\left[\xi_{i}, \xi_{i+1}\right)$ of size $\Delta \xi=2 \ell /(N+1)$. Under such notations, we have $\xi_{i}=-\ell+i \Delta \xi$, for $i=0, \ldots, N+1$. We denote by $w_{i}$ an approximation of $w\left(\xi_{i}\right), i=1, \ldots, N$. In fact, in order to deal with discrete boundary conditions, it is useful to enlarge the interval discretization from $[-\ell, \ell]$ to $[-\ell-2 \Delta \xi, \ell+2 \Delta \xi]$. As a consequence, the nodes of the discretization are defined by $\xi_{i}=-\ell+i \Delta \xi$, for $i=-2, \ldots, N+3$.

Next, for a given vector $\left(X_{i}\right)_{i=-2, \ldots, N+3}$, let us introduce the following discrete operators, for $i=1, \ldots, N$ :

$$
\begin{aligned}
& \overline{g(X)}_{i}^{\prime}=\frac{g\left(X_{i-2}\right)-8 g\left(X_{i-1}\right)+8 g\left(X_{i+1}\right)-g\left(X_{i+2}\right)}{12 \Delta \xi}, \\
& \bar{X}_{i}^{\prime \prime}=\frac{-X_{i-2}+16 X_{i-1}-30 X_{i}+16 X_{i+1}-X_{i+2}}{12 \Delta \xi^{2}} \\
& \bar{X}_{i}^{\prime \prime \prime}=\frac{X_{i-3}-8 X_{i-2}+13 X_{i-1}-13 X_{i+1}+8 X_{i+2}-X_{i+3}}{8 \Delta \xi^{3}},
\end{aligned}
$$

where $g(X)$ is a given smooth function. We immediately notice that, as soon as $U_{i}=U\left(\xi_{i}\right)$, where $U(\xi)$ denotes a smooth function, we get

$$
\begin{aligned}
& \overline{g(U)}_{i}^{\prime}=g(U)^{\prime}\left(\xi_{i}\right)+\mathcal{O}\left(\Delta \xi^{4}\right), \\
& \bar{U}_{i}^{\prime \prime}=U^{\prime \prime}\left(\xi_{i}\right)+\mathcal{O}\left(\Delta \xi^{4}\right), \\
& \bar{U}_{i}^{\prime \prime \prime}=U^{\prime \prime \prime}\left(\xi_{i}\right)+\mathcal{O}\left(\Delta \xi^{4}\right) .
\end{aligned}
$$

As a consequence, equipped with the above discrete operators, we state the finite difference scheme to approximate the solutions of (2.1) and (2.2) as follows:

$$
-\xi_{i} \bar{w}_{i}^{\prime}+\overline{f(w)}_{i}^{\prime}=\varepsilon \beta \bar{w}_{i}^{\prime \prime}+\varepsilon^{2} \gamma \bar{w}_{i}^{\prime \prime \prime}, \quad i=1, \ldots, N,
$$

supplemented by the following boundary conditions (for instance, see [20]):

$$
w_{-2}=w_{-1}=w_{0}=w_{L}, \quad w_{N+1}=w_{N+2}=w_{N+3}=w_{R} .
$$

We can justify this Dirichlet boundary condition choice according to [6], since, as soon as $\ell$ is large enough, the exact solution takes the expected left and right values. After [1] devoted to non-autonomous ODE problems, the Dirichlet boundary conditions are confirmed as suitable alternative. In order to avoid some confusion in the notations, $\bar{w}_{i}^{\prime}$ is defined by $(2.3)$ where we have set $g(w)=w$ and $\overline{f(w)}_{i}^{\prime}$ is also given by (2.3) but for $g(w)=f(w)$.

According to (2.6), the scheme (2.7) is of fourth-order of accuracy.

We are now able to state the existence of a vector $w^{\Delta}=\left(w_{i}\right)_{i=1, \ldots, N} \in \mathbb{R}^{N}$ solution of (2.7) and (2.8). 
TheOREm 1 (Existence result, scalar case). Let $\varepsilon>0$ be given and assume the existence of

$$
M_{f^{\prime}}:=\sup _{w \in \mathbb{R}}\left|f^{\prime}(w)\right| .
$$

Then there exists $\Delta \xi_{0} \leq \sqrt{\varepsilon \beta}$ depending on $\beta, \varepsilon$, $\ell$ and $M_{f^{\prime}}$ such that for $\Delta \xi \leq \Delta \xi_{0}$, there exists a solution $w^{\Delta}=\left(w_{i}\right)_{i=1, \ldots, N}$ to the scheme $(2.7)-(2.8)$.

From now on, let us underline that the above result does not depend on the interval length parameterized by $\ell$. However, in order to get a convergence to the expected solution of (1.1)-(1.2), the value of $\ell$ must be large enough such that the domain under consideration contains all the waves involved by the Riemann problem.

The rest of this section is dedicated to the proof of the above result. To address such an issue, we first reformulate the scheme (2.7) in order to deal with homogeneous Dirichlet boundary conditions instead of (2.8). In this way, we introduce the map $\mathcal{E}: w^{\Delta} \in \mathbb{R}^{N} \mapsto \mathcal{E}\left(w^{\Delta}\right) \in \mathbb{R}^{N}$ defined by

$$
\mathcal{E}\left(w^{\Delta}\right)_{i}=\varepsilon \beta \bar{w}_{i}^{\prime \prime}+\varepsilon^{2} \gamma \bar{w}_{i}^{\prime \prime \prime}+\xi_{i} \bar{w}_{i}^{\prime}-\overline{f(w)}_{i}^{\prime}, \quad i=1, \ldots, N,
$$

so that the scheme (2.7) rewrites

$$
\mathcal{E}\left(w^{\Delta}\right)=0 .
$$

For the sake of simplicity in the notations, we set $L: w^{\Delta} \in \mathbb{R}^{N} \mapsto L\left(w^{\Delta}\right) \in \mathbb{R}^{N}$, to denote the linear part within $\mathcal{E}$, given by

$$
L\left(w^{\Delta}\right)_{i}=\varepsilon \beta \bar{w}_{i}^{\prime \prime}+\varepsilon^{2} \gamma \bar{w}_{i}^{\prime \prime \prime}+\xi_{i} \bar{w}_{i}^{\prime}, \quad i=1, \ldots, N .
$$

Hence, for all $w^{\Delta} \in \mathbb{R}^{N}$, we have

$$
\mathcal{E}\left(w^{\Delta}\right)=L\left(w^{\Delta}\right)-{\overline{f\left(w^{\Delta}\right)}}^{\prime},
$$

where ${\overline{f\left(w^{\Delta}\right)}}^{\prime} \in \mathbb{R}^{N}$ is the vector constituted of ${\overline{f\left(w^{\Delta}\right)}}_{i}^{\prime}=\overline{f(w)}_{i}^{\prime}$ for $i=1, \ldots, N$.

Next, in order to recover the expected homogeneous Dirichlet conditions, we suggest to consider the new vector $\tilde{w}^{\Delta} \in \mathbb{R}^{N}$ defined by

$$
\tilde{w}^{\Delta}=w^{\Delta}-r^{\Delta},
$$

where $r^{\Delta}=\left(r\left(\xi_{i}\right)\right)_{i=1, \ldots, N}$. Here, $r(\xi)$ stands for the unique five-order polynomial function such that

$$
\begin{aligned}
& r\left(\xi_{-2}\right)=r\left(\xi_{-1}\right)=r\left(\xi_{0}\right)=w_{L}, \\
& r\left(\xi_{N+1}\right)=r\left(\xi_{N+2}\right)=r\left(\xi_{N+3}\right)=w_{R} .
\end{aligned}
$$

By construction, we directly have

$$
\tilde{w}_{-2}=\tilde{w}_{-1}=\tilde{w}_{0}=\tilde{w}_{N+1}=\tilde{w}_{N+2}=\tilde{w}_{N+3}=0,
$$

and thus the required homogeneous Dirichlet boundary conditions are recovered.

Next, let us reformulate the scheme (2.7), or equivalently (2.10), according to the new state vector $\tilde{w}^{\Delta}$. To do so, we introduce

$$
\begin{aligned}
\tilde{\mathcal{E}}\left(\tilde{w}^{\Delta}\right) & =\mathcal{E}\left(\tilde{w}^{\Delta}+r^{\Delta}\right), \\
& =L\left(\tilde{w}^{\Delta}\right)_{i}+L\left(r^{\Delta}\right)-{\overline{f\left(w^{\Delta}\right)}}^{\prime},
\end{aligned}
$$


where $w^{\Delta}=\tilde{w}^{\Delta}+r^{\Delta}$. One immediately recognizes a new equivalent formulation of the scheme (2.7) as follows:

$$
\tilde{\mathcal{E}}\left(\tilde{w}^{\Delta}\right)=0 .
$$

As a consequence, proving the existence of $w^{\Delta} \in \mathbb{R}^{N}$, solution of (2.7) and (2.8), is equivalent to proving the existence of $\tilde{w}^{\Delta} \in \mathbb{R}^{N}$, solution of (2.15) and (2.13). To address such an issue, we apply the following lemma (see [9, Section 9.1] for the proof), which is a consequence of the Brouwer's fixed point theorem.

Lemma 1 (Zeros of a vector field). Let $F: \mathbb{R}^{N} \rightarrow \mathbb{R}^{N}$ be a continuous function satisfying

$$
F(x) \cdot x \leq 0 \quad \text { if }\|x\|=k,
$$

for some $k>0$. Then there exists a point $x \in B(0, k)$ such that $F(x)=0$.

Since $f$ is assumed to be smooth, $\tilde{\mathcal{E}}$ is clearly continuous. As a consequence, according to the above lemma, Theorem 1 will be proved as soon as we establish the existence of a constant $k>0$ such that $\tilde{\mathcal{E}}\left(\tilde{w}^{\Delta}\right) \cdot \tilde{w}^{\Delta} \leq 0$ for any $\tilde{w}^{\Delta} \in \mathbb{R}^{N}$ whose Euclidean norm satisfies $\left\|\tilde{w}^{\Delta}\right\|=k$.

Now, we study the scalar product $\tilde{\mathcal{E}}\left(\tilde{w}^{\Delta}\right) \cdot \tilde{w}^{\Delta}$ which can be developed as follows:

$$
\tilde{\mathcal{E}}\left(\tilde{w}^{\Delta}\right) \cdot \tilde{w}^{\Delta}=L\left(\tilde{w}^{\Delta}\right) \cdot \tilde{w}^{\Delta}+L\left(r^{\Delta}\right) \cdot \tilde{w}^{\Delta}-\overline{f\left(w^{\Delta}\right)} \cdot \tilde{w}^{\Delta} .
$$

In order to apply Lemma 1, we need to exhibit suitable estimations of each term in the above identity. This is the purpose of the three following technical lemmas.

Lemma 2. Let $L$ be the linear map defined by (2.11). Then we have

$$
\begin{gathered}
L\left(\tilde{w}^{\Delta}\right) \cdot \tilde{w}^{\Delta}=-\frac{1}{12}\left(\frac{\varepsilon \beta}{\Delta \xi^{2}}-1\right) \sum_{i=-1}^{N}\left(\tilde{w}_{i+2}-2 \tilde{w}_{i+1}+\tilde{w}_{i}\right)^{2} \\
-\frac{\varepsilon \beta}{\Delta \xi^{2}} \sum_{i=0}^{N}\left(\tilde{w}_{i+1}-\tilde{w}_{i}\right)^{2}-\frac{1}{2} \sum_{i=1}^{N} \tilde{w}_{i}^{2} .
\end{gathered}
$$

Proof. Let us set

$$
S_{1}=\sum_{i=1}^{N} \overline{\tilde{w}}_{i}^{\prime \prime} \tilde{w}_{i}, \quad S_{2}=\sum_{i=1}^{N} \overline{\tilde{w}}_{i}^{\prime \prime \prime} \tilde{w}_{i} \quad \text { and } \quad S_{3}=\sum_{i=1}^{N} \xi_{i} \overline{\tilde{w}}_{i}^{\prime} \tilde{w}_{i}
$$

so that we get

$$
L\left(\tilde{w}^{\Delta}\right) \cdot \tilde{w}^{\Delta}=\varepsilon \beta S_{1}+\varepsilon^{2} \gamma S_{2}+S_{3} .
$$

Now, we study separately the sums $S_{1}, S_{2}$ and $S_{3}$. Concerning the first sum, with $\overline{\tilde{w}}_{i}^{\prime \prime}$ given by (2.4), arguing changes of indices and homogeneous boundary conditions (2.13), we get the following sequence of equalities:

$$
\begin{aligned}
& 12 \Delta \xi^{2} S_{1}=\sum_{i=1}^{N}\left(-\tilde{w}_{i-2}+16 \tilde{w}_{i-1}-30 \tilde{w}_{i}+16 \tilde{w}_{i+1}-\tilde{w}_{i+2}\right) \tilde{w}_{i} \\
& =-\sum_{i=1}^{N-2} \tilde{w}_{i} \tilde{w}_{i+2}+16 \sum_{i=1}^{N-1} \tilde{w}_{i} \tilde{w}_{i+1}-30 \sum_{i=1}^{N} \tilde{w}_{i}^{2}+16 \sum_{i=1}^{N-1} \tilde{w}_{i+1} \tilde{w}_{i}-\sum_{i=1}^{N-2} \tilde{w}_{i+2} \tilde{w}_{i} \\
& =-2 \sum_{i=1}^{N-2} \tilde{w}_{i+2} \tilde{w}_{i}+32 \sum_{i=1}^{N-1} \tilde{w}_{i+1} \tilde{w}_{i}-30 \sum_{i=1}^{N} \tilde{w}_{i}^{2}
\end{aligned}
$$


Similarly, arguing (2.5) together with changes of indices and (2.13), the sum $S_{2}$ reads

$$
\begin{aligned}
8 \Delta \xi^{3} S_{2}= & \sum_{i=1}^{N}\left(\tilde{w}_{i-3}-8 \tilde{w}_{i-2}+13 \tilde{w}_{i-1}-13 \tilde{w}_{i+1}+8 \tilde{w}_{i+2}-\tilde{w}_{i+3}\right) \tilde{w}_{i} \\
= & \sum_{i=1}^{N-3} \tilde{w}_{i} \tilde{w}_{i+3}-8 \sum_{i=1}^{N-2} \tilde{w}_{i} \tilde{w}_{i+2}+13 \sum_{i=1}^{N-1} \tilde{w}_{i} \tilde{w}_{i+1} \\
& \quad-13 \sum_{i=1}^{N-1} \tilde{w}_{i+1} \tilde{w}_{i}+8 \sum_{i=1}^{N-2} \tilde{w}_{i+2} \tilde{w}_{i}-\sum_{i=1}^{N-3} \tilde{w}_{i+3} \tilde{w}_{i} \\
= & 0 .
\end{aligned}
$$

Finally, concerning $S_{3}$, once again involving a change of indices and (2.13), from (2.3), we have

$$
\begin{aligned}
12 \Delta \xi S_{3} & =\sum_{i=1}^{N} \xi_{i}\left(\tilde{w}_{i-2}-8 \tilde{w}_{i-1}+8 \tilde{w}_{i+1}-\tilde{w}_{i+2}\right) \tilde{w}_{i} \\
& =\sum_{i=1}^{N-2} \xi_{i+2} \tilde{w}_{i} \tilde{w}_{i+2}-8 \sum_{i=1}^{N-1} \xi_{i+1} \tilde{w}_{i} \tilde{w}_{i+1}+8 \sum_{i=1}^{N-1} \xi_{i} \tilde{w}_{i+1} \tilde{w}_{i}-\sum_{i=1}^{N-2} \xi_{i} \tilde{w}_{i+2} \tilde{w}_{i} .
\end{aligned}
$$

Moreover, since $\xi_{i+2}-\xi_{i}=2 \Delta \xi$ and $\xi_{i+1}-\xi_{i}=\Delta \xi$, the above relation now reads

$$
12 \Delta \xi S_{3}=\sum_{i=1}^{N-2} 2 \Delta \xi \tilde{w}_{i+2} \tilde{w}_{i}-8 \sum_{i=1}^{N-1} \Delta \xi \tilde{w}_{i+1} \tilde{w}_{i} .
$$

We plug the three relations (2.21), (2.22) and (2.23) together in (2.20) to obtain

$$
\begin{aligned}
L\left(\tilde{w}^{\Delta}\right) \cdot \tilde{w}^{\Delta}=\frac{1}{6}( & \left.\frac{\varepsilon \beta}{\Delta \xi^{2}}-1\right) \sum_{i=1}^{N-2}\left(-\tilde{w}_{i} \tilde{w}_{i+2}\right) \\
& +\frac{2}{3}\left(\frac{4 \varepsilon \beta}{\Delta \xi^{2}}-1\right) \sum_{i=1}^{N-1} \tilde{w}_{i} \tilde{w}_{i+1}-\frac{5 \varepsilon \beta}{2 \Delta \xi^{2}} \sum_{i=1}^{N} \tilde{w}_{i}^{2} .
\end{aligned}
$$

Now, on the one hand, since we have for all $i=1, \ldots, N$

$$
\begin{aligned}
-\tilde{w}_{i} \tilde{w}_{i+2}=- & \frac{1}{2}\left(\left(\tilde{w}_{i+1}-\tilde{w}_{i}\right)-\left(\tilde{w}_{i+2}-\tilde{w}_{i+1}\right)\right)^{2}+\frac{1}{2}\left(\tilde{w}_{i+1}-\tilde{w}_{i}\right)^{2} \\
& +\frac{1}{2}\left(\tilde{w}_{i+2}-\tilde{w}_{i+1}\right)^{2}-\tilde{w}_{i} \tilde{w}_{i+1}-\tilde{w}_{i+1} \tilde{w}_{i+2}+\tilde{w}_{i+1}^{2}
\end{aligned}
$$

from the zero boundary conditions (2.13), we easily obtain

$$
\begin{aligned}
\sum_{i=1}^{N-2}\left(-\tilde{w}_{i} \tilde{w}_{i+2}\right)= & \sum_{i=-1}^{N}\left(-\tilde{w}_{i} \tilde{w}_{i+2}\right) \\
=- & \frac{1}{2} \sum_{i=-1}^{N}\left(\tilde{w}_{i+2}-2 \tilde{w}_{i+1}+\tilde{w}_{i}\right)^{2}+\sum_{i=0}^{N}\left(\tilde{w}_{i+1}-\tilde{w}_{i}\right)^{2} \\
& \quad-2 \sum_{i=0}^{N} \tilde{w}_{i} \tilde{w}_{i+1}+\sum_{i=1}^{N} \tilde{w}_{i}^{2} .
\end{aligned}
$$


On the other hand, because of (2.13), we have

$$
\begin{aligned}
\sum_{i=1}^{N-1} \tilde{w}_{i} \tilde{w}_{i+1} & =\sum_{i=0}^{N} \tilde{w}_{i} \tilde{w}_{i+1} \\
& =-\frac{1}{2} \sum_{i=0}^{N}\left(\tilde{w}_{i+1}-\tilde{w}_{i}\right)^{2}+\frac{1}{2} \sum_{i=0}^{N} \tilde{w}_{i+1}^{2}+\frac{1}{2} \sum_{i=0}^{N} \tilde{w}_{i}^{2} .
\end{aligned}
$$

Now, involving (2.26) and (2.27), we easily reformulate $L\left(\tilde{w}^{\Delta}\right) \cdot \tilde{w}^{\Delta}$, given by $(2.24)$, into the required form (2.18). The proof is thus achieved. $\square$

LEMma 3. Let $L$ be the linear map defined by (2.11). Then, there exists a constant $\Lambda>0$, which depends on $\Delta \xi, \ell, w_{L}$ and $w_{R}$, such that

$$
L\left(r^{\Delta}\right) \cdot \tilde{w}^{\Delta} \leq \Lambda \sqrt{\frac{2 \ell}{\Delta \xi}}\left\|\tilde{w}^{\Delta}\right\|
$$

Proof. Since $L$ is defined by (2.11), we have

$$
L\left(r^{\Delta}\right)_{i}=\varepsilon \beta \bar{r}_{i}^{\prime \prime}+\varepsilon^{2} \gamma \bar{r}_{i}^{\prime \prime \prime}+\xi_{i} \bar{r}_{i}^{\prime}
$$

where $\bar{r}_{i}^{\prime}, \bar{r}_{i}^{\prime \prime}$ and $\bar{r}_{i}^{\prime \prime \prime}$ are defined, with clear notations, by (2.3), (2.4) and (2.5) respectively.

On the one hand, since $r$ is a five-order polynomial, we have $\bar{r}_{i}^{\prime \prime}=r^{\prime \prime}\left(\xi_{i}\right)$ and $\bar{r}_{i}^{\prime \prime \prime}=r^{\prime \prime \prime}\left(\xi_{i}\right)$. Concerning $\bar{r}_{i}^{\prime}$, we easily get

$$
\bar{r}_{i}^{\prime}=r^{\prime}\left(\xi_{i}\right)+\frac{\Delta \xi^{4}}{5 !} r_{5}
$$

where $r_{5}$ is the constant value of the fifth-order derivative of $r$. As a consequence, we deduce that there exists a constant $\Lambda>0$, depending on $\Delta \xi, \ell, w_{L}$ and $w_{R}$, such that

$$
\left|L(r)_{i}\right| \leq \Lambda \quad \forall i=1, \ldots, N
$$

On the other hand, the Cauchy-Schwarz inequality directly leads to

$$
\sum_{i=1}^{N}\left|\tilde{w}_{i}\right| \leq \sqrt{N}\left\|\tilde{w}^{\Delta}\right\| \leq \sqrt{N+1}\left\|\tilde{w}^{\Delta}\right\|=\sqrt{\frac{2 \ell}{\Delta \xi}}\left\|\tilde{w}^{\Delta}\right\| .
$$

Since we have

$$
L\left(r^{\Delta}\right) \cdot \tilde{w}^{\Delta} \leq \sum_{i=1}^{N}\left|L(r)_{i}\right|\left|\tilde{w}_{i}\right|
$$

we get the required inequality (2.28) and the proof is achieved. $\square$

LEMMA 4. Let $f: \mathbb{R} \rightarrow \mathbb{R}$ be a given smooth function. Let us set

$$
M_{f^{\prime}}:=\sup _{\xi \in \mathbb{R}}\left|f^{\prime}(\xi)\right|
$$

We denote by $\mathcal{F}$ a primitive function of $f$, so that $\mathcal{F}^{\prime}=f$. Then we have

$$
{\overline{f\left(w^{\Delta}\right)}}^{\prime} \cdot \tilde{w}^{\Delta} \geq-\frac{97}{24 \Delta \xi} M_{f^{\prime}} \sum_{i=0}^{N}\left(\tilde{w}_{i+1}-\tilde{w}_{i}\right)^{2}-A_{0}
$$


where we have set

$$
\begin{aligned}
A_{0}= & \frac{1}{\Delta \xi}\left|\mathcal{F}\left(w_{R}\right)-\mathcal{F}\left(w_{L}\right)-f\left(w_{R}\right) w_{R}+f\left(w_{L}\right) w_{L}\right|+\frac{4 M_{f^{\prime}}}{\Delta \xi} \sum_{i=0}^{N}\left(r_{i+1}-r_{i}\right)^{2} \\
& +\frac{M_{f^{\prime}}}{24 \Delta \xi} \sum_{i=0}^{N}\left(r_{i+2}-7 r_{i+1}-7 r_{i}+r_{i-1}\right)^{2} \\
& +\frac{M_{f^{\prime}}}{12 \Delta \xi} \sum_{i=0}^{N}\left|r_{i+1}-r_{i}\right|\left|r_{i+2}-7 r_{i+1}-7 r_{i}+r_{i-1}\right| .
\end{aligned}
$$

Proof. For the sake of simplicity in the notations, we set

$$
S\left(w^{\Delta}, \tilde{w}^{\Delta}\right)=12 \Delta \xi \sum_{i=1}^{N} \overline{f(w)}_{i}^{\prime} \tilde{w}_{i} .
$$

Arguing (2.3) and a change of indices, we have

$$
\begin{aligned}
S\left(w^{\Delta}, \tilde{w}^{\Delta}\right) & =\sum_{i=1}^{N}\left(f\left(w_{i-2}\right)-8 f\left(w_{i-1}\right)+8 f\left(w_{i+1}\right)-f\left(w_{i+2}\right)\right) \tilde{w}_{i} \\
= & \sum_{i=-1}^{N-2} f\left(w_{i}\right) \tilde{w}_{i+2}-8 \sum_{i=0}^{N-1} f\left(w_{i}\right) \tilde{w}_{i+1}+8 \sum_{i=2}^{N+1} f\left(w_{i}\right) \tilde{w}_{i-1}-\sum_{i=3}^{N+2} f\left(w_{i}\right) \tilde{w}_{i-2} .
\end{aligned}
$$

Because of the zero boundary conditions (2.13), and adding and subtracting terms with $\tilde{w}_{i}$, we obtain

$$
\begin{aligned}
S\left(w^{\Delta}, \tilde{w}^{\Delta}\right)= & \sum_{i=-1}^{N} f\left(w_{i}\right)\left(\tilde{w}_{i+2}-\tilde{w}_{i}\right)-8 \sum_{i=0}^{N} f\left(w_{i}\right)\left(\tilde{w}_{i+1}-\tilde{w}_{i}\right) \\
& +8 \sum_{i=1}^{N+1} f\left(w_{i}\right)\left(\tilde{w}_{i-1}-\tilde{w}_{i}\right)-\sum_{i=1}^{N+2} f\left(w_{i}\right)\left(\tilde{w}_{i-2}-\tilde{w}_{i}\right) .
\end{aligned}
$$

Since $\tilde{w}^{\Delta}=w^{\Delta}-r^{\Delta}$, we split $S\left(w^{\Delta}, \tilde{w}^{\Delta}\right)$ as follows:

$$
S\left(w^{\Delta}, \tilde{w}^{\Delta}\right)=S\left(w^{\Delta}, w^{\Delta}\right)-S\left(w^{\Delta}, r^{\Delta}\right) .
$$

Concerning $S\left(w^{\Delta}, w^{\Delta}\right)$, once again arguing a change of indices, we get

$$
\begin{gathered}
S\left(w^{\Delta}, w^{\Delta}\right)=\sum_{i=-1}^{N} f\left(w_{i}\right)\left(w_{i+2}-w_{i}\right)-8 \sum_{i=0}^{N} f\left(w_{i}\right)\left(w_{i+1}-w_{i}\right) \\
\quad+8 \sum_{i=0}^{N} f\left(w_{i+1}\right)\left(w_{i}-w_{i+1}\right)-\sum_{i=-1}^{N} f\left(w_{i+2}\right)\left(w_{i}-w_{i+2}\right) \\
=\sum_{i=-1}^{N}\left(f\left(w_{i}\right)+f\left(w_{i+2}\right)\right)\left(w_{i+2}-w_{i}\right)-8 \sum_{i=0}^{N}\left(f\left(w_{i}\right)+f\left(w_{i+1}\right)\right)\left(w_{i+1}-w_{i}\right) .
\end{gathered}
$$


Now, let us introduce $\mathcal{F}$ a primitive of the function $f$. Usual Taylor's expansions immediately give

$$
\begin{aligned}
\mathcal{F}\left(w_{i+1}\right) & =\mathcal{F}\left(w_{i}\right)+\left(w_{i+1}-w_{i}\right) f\left(w_{i}\right)+\left(w_{i+1}-w_{i}\right)^{2} f^{\prime}\left(\theta_{1}\right), \\
\mathcal{F}\left(w_{i}\right) & =\mathcal{F}\left(w_{i+1}\right)-\left(w_{i+1}-w_{i}\right) f\left(w_{i+1}\right)+\left(w_{i+1}-w_{i}\right)^{2} f^{\prime}\left(\theta_{2}\right), \\
\mathcal{F}\left(w_{i+2}\right) & =\mathcal{F}\left(w_{i}\right)+\left(w_{i+2}-w_{i}\right) f\left(w_{i}\right)+\left(w_{i+2}-w_{i}\right)^{2} f^{\prime}\left(\theta_{3}\right), \\
\mathcal{F}\left(w_{i}\right) & =\mathcal{F}\left(w_{i+2}\right)-\left(w_{i+2}-w_{i}\right) f\left(w_{i+2}\right)+\left(w_{i+2}-w_{i}\right)^{2} f^{\prime}\left(\theta_{4}\right),
\end{aligned}
$$

where we have imposed

$$
\begin{aligned}
& \theta_{1}, \theta_{2} \in\left(\min \left(w_{i}, w_{i+1}\right), \max \left(w_{i}, w_{i+1}\right)\right), \\
& \theta_{3}, \theta_{4} \in\left(\min \left(w_{i}, w_{i+2}\right), \max \left(w_{i}, w_{i+2}\right)\right) .
\end{aligned}
$$

Then, we deduce the following equalities:

$$
\begin{aligned}
& \sum_{i=0}^{N}\left(f\left(w_{i}\right)+f\left(w_{i+1}\right)\right)\left(w_{i+1}-w_{i}\right)= \\
& 2\left(\mathcal{F}\left(w_{R}\right)-\mathcal{F}\left(w_{L}\right)\right)+\sum_{i=0}^{N}\left(w_{i+1}-w_{i}\right)^{2}\left(f^{\prime}\left(\theta_{2}\right)-f^{\prime}\left(\theta_{1}\right)\right) \\
& \sum_{i=-1}^{N}\left(f\left(w_{i}\right)+f\left(w_{i+2}\right)\right)\left(w_{i+2}-w_{i}\right)= \\
& 4\left(\mathcal{F}\left(w_{R}\right)-\mathcal{F}\left(w_{L}\right)\right)+\sum_{i=-1}^{N}\left(w_{i+2}-w_{i}\right)^{2}\left(f^{\prime}\left(\theta_{4}\right)-f^{\prime}\left(\theta_{3}\right)\right)
\end{aligned}
$$

so that $S\left(w^{\Delta}, w^{\Delta}\right)$ now writes

$$
\begin{gathered}
S\left(w^{\Delta}, w^{\Delta}\right)=-12\left(\mathcal{F}\left(w_{R}\right)-\mathcal{F}\left(w_{L}\right)\right)+\sum_{i=-1}^{N}\left(w_{i+2}-w_{i}\right)^{2}\left(f^{\prime}\left(\theta_{4}\right)-f^{\prime}\left(\theta_{3}\right)\right) \\
-8 \sum_{i=0}^{N}\left(w_{i+1}-w_{i}\right)^{2}\left(f^{\prime}\left(\theta_{2}\right)-f^{\prime}\left(\theta_{1}\right)\right) .
\end{gathered}
$$

Concerning $S\left(w^{\Delta}, r^{\Delta}\right)$, according to (2.31), we have

$$
\begin{aligned}
S\left(w^{\Delta}, r^{\Delta}\right)= & \sum_{i=-1}^{N} f\left(w_{i}\right)\left(r_{i+2}-r_{i}\right)-8 \sum_{i=0}^{N} f\left(w_{i}\right)\left(r_{i+1}-r_{i}\right) \\
& +8 \sum_{i=1}^{N+1} f\left(w_{i}\right)\left(r_{i-1}-r_{i}\right)-\sum_{i=1}^{N+2} f\left(w_{i}\right)\left(r_{i-2}-r_{i}\right) .
\end{aligned}
$$

Next, involving the boundary conditions (2.12), the above relation now reads

$$
\begin{gathered}
S\left(w^{\Delta}, r^{\Delta}\right)=\sum_{i=-1}^{N} f\left(w_{i}\right) r_{i+2}-8 \sum_{i=0}^{N} f\left(w_{i}\right) r_{i+1}+8 \sum_{i=1}^{N+1} f\left(w_{i}\right) r_{i-1} \\
-\sum_{i=1}^{N+2} f\left(w_{i}\right) r_{i-2}+6 f\left(w_{L}\right) w_{L}-6 f\left(w_{R}\right) w_{R} .
\end{gathered}
$$


We reformulate the sums to make appear terms $f\left(w_{i}\right)-f\left(w_{i+1}\right)$ as follows:

$$
\begin{aligned}
S\left(w^{\Delta}, r^{\Delta}\right)= & 6 f\left(w_{L}\right) w_{L}-6 f\left(w_{R}\right) w_{R}+\left(\sum_{i=-1}^{N} f\left(w_{i}\right) r_{i+2}-\sum_{i=-1}^{N-1} f\left(w_{i+1}\right) r_{i+2}\right) \\
& -7\left(\sum_{i=0}^{N} f\left(w_{i}\right) r_{i+1}-\sum_{i=-1}^{N-1} f\left(w_{i+1}\right) r_{i+1}\right) \\
& -7\left(\sum_{i=0}^{N} f\left(w_{i}\right) r_{i}-\sum_{i=0}^{N} f\left(w_{i+1}\right) r_{i}\right) \\
& +\left(\sum_{i=1}^{N+1} f\left(w_{i}\right) r_{i-1}-\sum_{i=0}^{N+1} f\left(w_{i+1}\right) r_{i-1}\right) .
\end{aligned}
$$

Once again, involving (2.12), we get

$$
\begin{aligned}
& S\left(w^{\Delta}, r^{\Delta}\right)=12\left(f\left(w_{L}\right) w_{L}-f\left(w_{R}\right) w_{R}\right) \\
& \quad+\sum_{i=0}^{N}\left(f\left(w_{i}\right)-f\left(w_{i+1}\right)\right)\left(r_{i+2}-7 r_{i+1}-7 r_{i}+r_{i-1}\right) .
\end{aligned}
$$

From (2.32) and (2.33), we deduce

$$
\begin{aligned}
S\left(w^{\Delta}, \tilde{w}^{\Delta}\right)=-12 & \left(\mathcal{F}\left(w_{R}\right)-\mathcal{F}\left(w_{L}\right)-f\left(w_{R}\right) w_{R}+f\left(w_{L}\right) w_{L}\right) \\
& +\sum_{i=-1}^{N}\left(w_{i+2}-w_{i}\right)^{2}\left(f^{\prime}\left(\theta_{4}\right)-f^{\prime}\left(\theta_{3}\right)\right) \\
& -8 \sum_{i=0}^{N}\left(w_{i+1}-w_{i}\right)^{2}\left(f^{\prime}\left(\theta_{2}\right)-f^{\prime}\left(\theta_{1}\right)\right) \\
& +\sum_{i=0}^{N} \frac{f\left(w_{i+1}\right)-f\left(w_{i}\right)}{w_{i+1}-w_{i}}\left(w_{i+1}-w_{i}\right)\left(r_{i+2}-7 r_{i+1}-7 r_{i}+r_{i-1}\right) .
\end{aligned}
$$

Since we have

$$
\left|\frac{f\left(w_{i+1}\right)-f\left(w_{i}\right)}{w_{i+1}-w_{i}}\right| \leq M_{f^{\prime}}
$$

and

$$
\begin{aligned}
\left(w_{i+1}-w_{i}\right) & \left(r_{i+2}-7 r_{i+1}-7 r_{i}+r_{i-1}\right) \leq \\
& \frac{1}{2}\left(\tilde{w}_{i+1}-\tilde{w}_{i}\right)^{2}+\frac{1}{2}\left(r_{i+2}-7 r_{i+1}-7 r_{i}+r_{i-1}\right)^{2} \\
& +\left(r_{i+1}-r_{i}\right)\left(r_{i+2}-7 r_{i+1}-7 r_{i}+r_{i-1}\right)
\end{aligned}
$$


we finally get

$$
\begin{aligned}
S\left(w^{\Delta}, r^{\Delta}\right) \geq & -12\left|\mathcal{F}\left(w_{R}\right)-\mathcal{F}\left(w_{L}\right)-f\left(w_{R}\right) w_{R}+f\left(w_{L}\right) w_{L}\right| \\
& -2 M_{f^{\prime}} \sum_{i=-1}^{N}\left(w_{i+2}-w_{i}\right)^{2}-16 M_{f^{\prime}} \sum_{i=0}^{N}\left(w_{i+1}-w_{i}\right)^{2} \\
& -\frac{M_{f^{\prime}}}{2} \sum_{i=0}^{N}\left(\tilde{w}_{i+1}-\tilde{w}_{i}\right)^{2}-\frac{M_{f^{\prime}}}{2} \sum_{i=0}^{N}\left(r_{i+2}-7 r_{i+1}-7 r_{i}+r_{i-1}\right)^{2} \\
& -M_{f^{\prime}} \sum_{i=0}^{N}\left|r_{i+1}-r_{i}\right|\left|r_{i+2}-7 r_{i+1}-7 r_{i}+r_{i-1}\right| .
\end{aligned}
$$

Now, arguing the following inequalities:

$$
\begin{aligned}
\sum_{i=-1}^{N}\left(w_{i+2}-w_{i}\right)^{2} & \leq 4 \sum_{i=0}^{N}\left(w_{i+1}-w_{i}\right)^{2} \\
& \leq 2 \sum_{i=0}^{N+1}\left(w_{i+1}-w_{i}\right)^{2}+2 \sum_{i=-1}^{N}\left(w_{i+1}-w_{i}\right)^{2} \\
& \leq 8 \sum_{i=0}^{N}\left(\tilde{w}_{i+1}-\tilde{w}_{i}\right)^{2}+8 \sum_{i=0}^{N}\left(r_{i+1}-r_{i}\right)^{2}
\end{aligned}
$$

we directly obtain the expected estimation (2.29). The proof is thus completed.

Equipped with the above three lemmas, we are now able to demonstrate Theorem 1.

Proof. Gathering estimates (2.18), (2.28) and (2.29) provides

$$
\begin{aligned}
\tilde{\mathcal{E}}\left(\tilde{w}^{\Delta}\right) \cdot \tilde{w}^{\Delta} \leq & -\frac{1}{12}\left(\frac{\varepsilon \beta}{\Delta \xi^{2}}-1\right) \sum_{i=-1}^{N}\left(\tilde{w}_{i+2}-2 \tilde{w}_{i+1}+\tilde{w}_{i}\right)^{2}-\frac{\varepsilon \beta}{\Delta \xi^{2}} \sum_{i=0}^{N}\left(\tilde{w}_{i+1}-\tilde{w}_{i}\right)^{2} \\
& -\frac{1}{2}\left\|\tilde{w}^{\Delta}\right\|^{2}+\frac{97 M_{f^{\prime}}}{24 \Delta \xi} \sum_{i=0}^{N}\left(\tilde{w}_{i+1}-\tilde{w}_{i}\right)^{2}+A_{0}+\sqrt{\frac{2 \ell}{\Delta \xi}}\left\|\tilde{w}^{\Delta}\right\|
\end{aligned}
$$

where $A_{0}$ is given by $(2.30)$. Now, since $\tilde{w}^{\Delta}$ satisfies zero Dirichlet boundary conditions, the discrete Poincaré inequality can be applied. As a consequence, we have

$$
\sum_{i=-1}^{N}\left|\tilde{w}_{i+2}-2 \tilde{w}_{i+1}+\tilde{w}_{i}\right|^{2} \geq \frac{\Delta \xi^{2}}{4(2 \ell)^{2}} \sum_{i=0}^{N}\left|\tilde{w}_{i+1}-\tilde{w}_{i}\right|^{2},
$$

which leads to, as soon as $\Delta \xi^{2} \leq \varepsilon \beta$,

$$
\tilde{\mathcal{E}}\left(\tilde{w}^{\Delta}\right) \cdot \tilde{w}^{\Delta} \leq-\alpha \sum_{i=0}^{N}\left(\tilde{w}_{i+1}-\tilde{w}_{i}\right)^{2}-\frac{1}{2}\left\|\tilde{w}^{\Delta}\right\|^{2}+A_{0}+\sqrt{\frac{2 \ell}{\Delta \xi}}\left\|\tilde{w}^{\Delta}\right\|,
$$

where we have set

$$
\alpha=\frac{1}{12}\left(\frac{\varepsilon \beta}{\Delta \xi^{2}}-1\right) \frac{\Delta \xi^{2}}{4(2 \ell)^{2}}+\frac{\varepsilon \beta}{\Delta \xi^{2}}-\frac{97 M_{f^{\prime}}}{24 \Delta \xi}
$$


We remark that $\alpha$ tends to $+\infty$ as $\Delta \xi$ tends to 0 . Then, there exists $\Delta \xi_{0}>0$ depending only on $\ell, \varepsilon, \beta$ and $M_{f^{\prime}}$ such that for all $\Delta \xi \leq \Delta \xi_{0}$, we have $\alpha \geq 0$.

Once again, by the discrete Poincaré inequality applied to $\tilde{w}^{\Delta} \in \mathbb{R}^{N}$, we have

$$
\sum_{i=0}^{N}\left(\tilde{w}_{i+1}-\tilde{w}_{i}\right)^{2} \geq \frac{\Delta \xi^{2}}{(2 \ell)^{2}}\left\|\tilde{w}^{\Delta}\right\|^{2}
$$

to rewrite the inequality (2.35) as follows:

$$
\tilde{\mathcal{E}}\left(\tilde{w}^{\Delta}\right) \cdot \tilde{w}^{\Delta} \leq-\left(\frac{1}{2}+\alpha \frac{\Delta \xi^{2}}{(2 \ell)^{2}}\right)\left\|\tilde{w}^{\Delta}\right\|^{2}+\sqrt{\frac{2 \ell}{\Delta \xi}}\left\|\tilde{w}^{\Delta}\right\|+A_{0} .
$$

The right-hand side of the above inequality is a second-order polynomial function of $\left\|\tilde{w}^{\Delta}\right\|$ with negative leading order. Thus there exists $k>0$ such that if $\left\|\tilde{w}^{\Delta}\right\|=k$, then $\tilde{\mathcal{E}}\left(\tilde{w}^{\Delta}\right) \cdot \tilde{w}^{\Delta} \leq 0$. Lemma 1 then implies existence of $\tilde{w}^{\Delta}$ such that $\tilde{\mathcal{E}}\left(\tilde{w}^{\Delta}\right)=0$, and then existence of $w^{\Delta}$ solution to the scheme (2.7) and (2.8), which concludes the proof.

3. A p-system like model. In this section, a $2 \times 2$ diffusive-dispersive system, introduced by Joseph and LeFloch in [11], is considered. This model reads

$$
\left\{\begin{array}{l}
\partial_{t} w^{\varepsilon}-\partial_{x} v^{\varepsilon}=0, \quad x \in \mathbb{R}, t>0 \\
\partial_{t} v^{\varepsilon}-\partial_{x} p\left(w^{\varepsilon}\right)=\varepsilon t \beta \partial_{x x} v^{\varepsilon}+\varepsilon^{2} t^{2} \gamma \partial_{x x x} w^{\varepsilon},
\end{array}\right.
$$

where $p: \mathbb{R} \rightarrow \mathbb{R}$ is a given smooth function. From now on, let us note that we do not impose any restriction on $p^{\prime}$. In other words, in the present work, we do not enforce the first-order extracted system from (3.1) to be hyperbolic.

Since we are interested in self-similar solutions, we adopt the following change of variables:

$$
w^{\varepsilon}(x, t)=w(\xi) \quad \text { and } \quad v^{\varepsilon}(x, t)=v(\xi) \quad \text { with } \xi=\frac{x}{t},
$$

to get the following ODE system governing the unknown ${ }^{t}(v, w)$ :

$$
\left\{\begin{array}{l}
-\xi w^{\prime}-v^{\prime}=0, \\
-\xi v^{\prime}-p(w)^{\prime}=\varepsilon \beta v^{\prime \prime}+\varepsilon^{2} \gamma w^{\prime \prime \prime} .
\end{array}\right.
$$

According to (1.6), this system is supplemented by boundary conditions as follows:

$$
(v, w)(-\ell)=\left(v_{L}, w_{L}\right) \quad \text { and } \quad(v, w)(\ell)=\left(v_{R}, w_{R}\right),
$$

with $\ell>0$ large enough.

Let us underline that $w$ is governed by a nonlinear equation independent of $v$. Indeed, since $v^{\prime}=-\xi w^{\prime}$, we immediately obtain the following equation satisfied by $w$ :

$$
\left(\xi^{2}+\varepsilon \beta\right) w^{\prime}-p(w)^{\prime}=-\varepsilon \beta \xi w^{\prime \prime}+\varepsilon^{2} \gamma w^{\prime \prime \prime} .
$$

It is worth noticing that the above scalar ODE governing $w$, when supplemented by the boundary conditions

$$
w(-\ell)=w_{L} \quad \text { and } \quad w(\ell)=w_{R}
$$


contains the full structure of the expected Riemann solution.

After the work by Joseph and LeFloch in [11], the equation (3.3) must be studied into the two regions $[-\ell, 0)$ and $(0, \ell]$ separately. In fact, in [11], the analysis of $(3.3)$ introduces some degeneracy for $\xi=0$. From a numerical point of view, the situation turns out to be similar. More precisely, according to the numerical analysis performed to study the scheme (2.7), we understand that the viscous term is of prime importance. In the equation (3.3), this viscous term is governed by $\varepsilon \beta \xi$. It is thus clear that the viscosity vanishes as soon as $\xi=0$. As a consequence, to avoid $\xi=0$, we follow ideas introduced in [11] and we discretize (3.3) over the two intervals $\left[-\ell,-\xi^{\star}\right]$ and $\left[\xi^{\star}, \ell\right]$ separately where $\xi^{\star}>0$ is a given constant small enough. Now, we must equip (3.3) with suitable boundary conditions to be stated at $-\xi^{\star}$ and $\xi^{\star}$. As soon as $\xi^{\star}$ is small enough, we can assume (see [11]) that

$$
w\left(-\xi^{\star}\right)=w^{\star} \quad \text { and } \quad w\left(\xi^{\star}\right)=w^{\star},
$$

where $\xi^{\star}$ and $w^{\star}$ have to be determined. At this level, we assume that the state $w^{\star} \in \mathbb{R}$ is given, so that we can propose relevant discretization of (3.3) over $\left[-\ell,-\xi^{\star}\right]$ and $\left[\xi^{\star}, \ell\right]$ with boundary conditions given by (3.4) and (3.5). Next, we will detail the procedure to evaluate $w^{\star}$.

We present here the approximation on the right interval $\left[\xi^{\star}, \ell\right]$. The results are similar on the left interval $\left[-\ell,-\xi^{\star}\right]$ and they are left to the reader. We adopt a discretization of the interval $\left[\xi^{\star}, \ell\right]$, made of $N+1$ cells $\left[\xi_{i}, \xi_{i+1}\right)$ of constant size $\Delta \xi=\left(\ell-\xi^{\star}\right) /(N+1)$ so that $\xi_{i}=\xi^{\star}+i \Delta \xi$. In fact, in order to correctly prescribe the boundary conditions at the discrete level, this interval is enlarged to consider the domain $\left[\xi^{\star}-2 \Delta \xi, \ell+2 \Delta \xi\right]$. As a consequence, we are considering a sequence of nodes $\xi_{i}$ for $i=-2, \ldots, N+3$.

We denote by $w_{i}$ an approximation of $w\left(\xi_{i}\right)$ for $i=1, \cdots, N$ and we adopt the following fourth-order finite difference scheme:

$$
\left(\xi_{i}^{2}+\varepsilon \beta\right) \bar{w}_{i}^{\prime}-\overline{p(w)}_{i}^{\prime}=-\varepsilon \beta \xi_{i} \bar{w}_{i}^{\prime \prime}+\varepsilon^{2} \gamma \bar{w}_{i}^{\prime \prime \prime}, \quad i=1, \cdots, N,
$$

where $\bar{w}_{i}^{\prime}, \bar{w}_{i}^{\prime \prime}, \bar{w}_{i}^{\prime \prime \prime}$ are defined, with clear notations, by (2.3), (2.4) and (2.5). This scheme is completed with the following boundary conditions (for instance, see [20]):

$$
w_{-2}=w_{-1}=w_{0}=w^{\star}, \quad w_{N+1}=w_{N+2}=w_{N+3}=w_{R} .
$$

Now, by solving (3.6) and (3.7), we get $w^{\Delta}\left(w^{\star}, w_{R}\right) \in \mathbb{R}^{N}$ to approximate the solution of (3.3) with boundary conditions given by

$$
w\left(\xi^{\star}\right)=w^{\star} \quad \text { and } \quad w(\ell)=w_{R} .
$$

Similarly, we denote by $w^{\Delta}\left(w_{L}, w^{\star}\right)$ the approximated solution of (3.3) with boundary conditions given by

$$
w(-\ell)=w_{L} \quad \text { and } \quad w\left(-\xi^{\star}\right)=w^{\star} .
$$

Next, we have to determine $w^{\star}$. To address such an issue, let us emphasize that $w$, solution of (3.1), is governed by a conservation law. As a consequence, the total mass of $w$ must be conserved. At time $t=0$, the initial mass of $w$ is given by

$$
M_{0}=\ell\left(w_{L}+w_{R}\right)
$$


while the total mass of the approximated solution $w^{\Delta}$ depends on $w^{\star}$ and reads

$$
M\left(w^{\star}\right)=\Delta \xi w_{L}+\sum_{i=1}^{N} \Delta \xi w_{i}\left(w_{L}, w^{\star}\right)+2 \xi^{\star} w^{\star}+\sum_{i=1}^{N} \Delta \xi w_{i}\left(w^{\star}, w_{R}\right)+\Delta \xi w_{R}
$$

Then, $w^{\star}$ must be solution of the following nonlinear equation:

$$
M\left(w^{\star}\right)=M_{0} .
$$

Here, we assume that the mass function $M: \mathbb{R} \rightarrow \mathbb{R}$ is continuous, eventually monotone. To approximate the solution of the above equation, we adopt a dichotomy technique. First, we fix two initial values, $w_{L}^{\text {init }}$ and $w_{R}^{\text {init }}$, to compute $M\left(w_{L}^{\text {init }}\right)$ and $M\left(w_{R}^{\text {init }}\right)$. These two initial mass values must be chosen such that we have

$$
\min \left(M\left(w_{L}^{\text {init }}\right), M\left(w_{R}^{\text {init }}\right)\right) \leq M_{0} \leq \max \left(M\left(w_{L}^{\text {init }}\right), M\left(w_{R}^{\text {init }}\right)\right) .
$$

From a practical point of view, we underline that, in general, it suffices to initialize the process with $w_{L}^{\text {init }}=w_{L}$ and $w_{R}^{\text {init }}=w_{R}$.

Now, we initialize the dichotomy algorithm as follows:

$$
\left(w_{\text {inf }}, w_{\text {sup }}\right)=\left\{\begin{array}{l}
\left(w_{L}, w_{R}\right) \text { if } M\left(w_{L}\right)<M\left(w_{R}\right), \\
\left(w_{R}, w_{L}\right) \text { elsewhere }
\end{array} \quad w_{0}^{\star}=\frac{1}{2}\left(w_{L}+w_{R}\right) .\right.
$$

Then, for iterations $k \geq 1$, we compute the left and right solutions $w^{\Delta}\left(w_{L}, w_{k-1}^{\star}\right)$ and $w^{\Delta}\left(w_{k-1}^{\star}, w_{R}\right)$ and we deduce $M_{k}=M\left(w_{k-1}^{\star}\right)$ by (3.8). If $M_{k}<M_{0}$ then $w_{\text {inf }}=$ $w_{k-1}^{\star}$, else $w_{\text {sup }}=w_{k-1}^{\star}$, and we compute the new iterate value $w_{k}^{\star}=\frac{1}{2}\left(w_{\text {inf }}+w_{\text {sup }}\right)$. This achieves the presentation of the scheme to approximate the solutions of (3.3) and (3.4).

The objective now is to establish the existence of a vector $w^{\Delta}=\left(w_{i}\right)_{i=1, \ldots, N}$, solution of (3.6) completed with the boundary conditions (3.7).

Theorem 2 (Existence result, system case). Let $\varepsilon>0$ be given and $\xi^{\star}>2 \Delta \xi$. Assume the existence of

$$
M_{p^{\prime}}:=\sup _{w \in \mathbb{R}}\left|p^{\prime}(w)\right| .
$$

Then there exists $\Delta \xi_{0} \leq \sqrt{\varepsilon \beta}$ depending on $\beta, \varepsilon, \ell$, $\xi^{\star}$ and $M_{p^{\prime}}$ such that for $\Delta \xi \leq \Delta \xi_{0}$, there exists a solution $w^{\Delta}=\left(w_{i}\right)_{i=1, \ldots, N}$ to the scheme (3.6) with the boundary conditions (3.7).

For the sake of simplicity in the notations, let us introduce $\mathcal{E}: w^{\Delta} \in \mathbb{R}^{N} \mapsto$ $\mathcal{E}\left(w^{\Delta}\right) \in \mathbb{R}^{N}$, defined by

$$
\mathcal{E}\left(w^{\Delta}\right)_{i}=L\left(w^{\Delta}\right)_{i}-{\overline{p\left(w^{\Delta}\right)_{i}^{\prime}}}^{\prime}, \quad i=1, \ldots, N,
$$

where $L: w^{\Delta} \in \mathbb{R}^{N} \mapsto L\left(w^{\Delta}\right) \in \mathbb{R}^{N}$ is a linear map given as follows:

$$
L\left(w^{\Delta}\right)_{i}=\varepsilon \beta \xi_{i} \bar{w}_{i}^{\prime \prime}-\varepsilon^{2} \gamma \bar{w}_{i}^{\prime \prime \prime}+\left(\xi_{i}^{2}+\varepsilon \beta\right) \bar{w}_{i}^{\prime}, \quad i=1, \ldots, N .
$$

As a consequence, the scheme (3.6) now reads

$$
\mathcal{E}\left(w^{\Delta}\right)=0 .
$$


Next, in order to relevantly deal with the nonhomogeneous Dirichlet boundary conditions (3.7), we introduce the unique five-order polynomial function, denoted by $r(\xi)$, such that

$$
\begin{aligned}
& r\left(\xi_{-2}\right)=r\left(\xi_{-1}\right)=r\left(\xi_{0}\right)=w^{\star}, \\
& r\left(\xi_{N+1}\right)=r\left(\xi_{N+2}\right)=r\left(\xi_{N+3}\right)=w_{R} .
\end{aligned}
$$

Then, we define the vector $r^{\Delta}=\left(r_{i}\right)_{i=1, \ldots, N}$ where $r_{i}=r\left(\xi_{i}\right)$, to introduce $\tilde{w}^{\Delta}=$ $w^{\Delta}-r^{\Delta} \in \mathbb{R}^{N}$ which is clearly supplemented by homogeneous Dirichlet boundary conditions (2.13). Now, let us introduce $\tilde{\mathcal{E}}: \mathbb{R}^{N} \rightarrow \mathbb{R}^{N}$ defined as follows:

$$
\begin{aligned}
\tilde{\mathcal{E}}\left(\tilde{w}^{\Delta}\right)_{i} & =\mathcal{E}\left(\tilde{w}^{\Delta}+r^{\Delta}\right)_{i}, \\
& =L\left(\tilde{w}^{\Delta}\right)_{i}+L\left(r^{\Delta}\right)_{i}-{\overline{p\left(w^{\Delta}\right.}}_{i}^{\prime}, \quad i=1, \ldots, N,
\end{aligned}
$$

so that the discrete problem (3.6) is then equivalent to find $\tilde{w}^{\Delta} \in \mathbb{R}^{N}$ such that $\tilde{\mathcal{E}}\left(\tilde{w}^{\Delta}\right)=0$.

According to Lemma 1 , the existence of $\tilde{w}^{\Delta}$ such that $\tilde{\mathcal{E}}\left(\tilde{w}^{\Delta}\right)=0$ is established as soon as we prove the existence of a constant $k$ such that $\tilde{\mathcal{E}}\left(\tilde{w}^{\Delta}\right) \cdot \tilde{w}^{\Delta} \leq 0$ for all $\tilde{w}^{\Delta} \in \mathbb{R}^{N}$ with $\left\|\tilde{w}^{\Delta}\right\|=k$.

Since we have

$$
\tilde{\mathcal{E}}\left(\tilde{w}^{\Delta}\right) \cdot \tilde{w}^{\Delta}=L\left(\tilde{w}^{\Delta}\right) \cdot \tilde{w}^{\Delta}+L\left(r^{\Delta}\right) \cdot \tilde{w}^{\Delta}-{\overline{p\left(w^{\Delta}\right)}}^{\prime} \cdot \tilde{w}^{\Delta},
$$

we now study separately each scalar product involved within the above relation.

Lemma 5. Let $L$ be the linear map defined by (3.10). Then we have

$$
\begin{aligned}
L\left(\tilde{w}^{\Delta}\right) \cdot \tilde{w}^{\Delta}=-\frac{1}{6}\left(\frac{\varepsilon \beta}{2 \Delta \xi^{2}}\right. & -1) \sum_{i=-1}^{N} \xi_{i+1}\left(\tilde{w}_{i+2}-2 \tilde{w}_{i+1}+\tilde{w}_{i}\right)^{2} \\
& -\frac{\varepsilon \beta}{\Delta \xi^{2}} \sum_{i=0}^{N}\left(\xi_{i}+\frac{\Delta \xi}{2}\right)\left(\tilde{w}_{i+1}-\tilde{w}_{i}\right)^{2}-\sum_{i=1}^{N} \xi_{i} \tilde{w}_{i}^{2} .
\end{aligned}
$$

Proof. For the sake of clarity in the forthcoming developments, we set

$$
S_{1}=\sum_{i=1}^{N} \xi_{i} \overline{\widetilde{w}}_{i}^{\prime \prime} \tilde{w}_{i}, \quad S_{2}=\sum_{i=1}^{N} \overline{\tilde{w}}_{i}^{\prime \prime \prime} \tilde{w}_{i}, \quad \text { and } \quad S_{3}=\sum_{i=1}^{N}\left(\xi_{i}^{2}+\varepsilon \beta\right) \overline{\tilde{w}}_{i}^{\prime} \tilde{w}_{i},
$$

in order to reformulate $L\left(\tilde{w}^{\Delta}\right) \cdot \tilde{w}^{\Delta}$ as follows:

$$
L\left(\tilde{w}^{\Delta}\right) \cdot \tilde{w}^{\Delta}=\varepsilon \beta S_{1}-\varepsilon^{2} \gamma S_{2}+S_{3} .
$$

From now on, one may recognize in $S_{2}$ the same sum as the one introduced in (2.19). As a consequence, according to the sequence of equalities (2.22), we immediately get $S_{2}=0$. 
Concerning $S_{1}$, we have

$$
\begin{aligned}
12 \Delta \xi^{2} S_{1}= & \sum_{i=1}^{N}\left(-\tilde{w}_{i-2}+16 \tilde{w}_{i-1}-30 \tilde{w}_{i}+16 \tilde{w}_{i+1}-\tilde{w}_{i+2}\right) \xi_{i} \tilde{w}_{i} \\
=- & \sum_{i=1}^{N-2} \tilde{w}_{i} \xi_{i+2} \tilde{w}_{i+2}+16 \sum_{i=1}^{N-1} \tilde{w}_{i} \xi_{i+1} \tilde{w}_{i+1}-30 \sum_{i=1}^{N} \xi_{i} \tilde{w}_{i}^{2} \\
& \quad+16 \sum_{i=1}^{N-1} \tilde{w}_{i+1} \xi_{i} \tilde{w}_{i}-\sum_{i=1}^{N-2} \tilde{w}_{i+2} \xi_{i} \tilde{w}_{i} \\
=- & \sum_{i=1}^{N-2}\left(2 \xi_{i}+2 \Delta \xi\right) \tilde{w}_{i+2} \tilde{w}_{i}+16 \sum_{i=1}^{N-1}\left(2 \xi_{i}+\Delta \xi\right) \tilde{w}_{i+1} \tilde{w}_{i}-30 \sum_{i=1}^{N} \xi_{i} \tilde{w}_{i}^{2} .
\end{aligned}
$$

Next, the sum $S_{3}$ writes as follows:

$$
\begin{aligned}
12 \Delta \xi S_{3}= & \sum_{i=1}^{N}\left(\xi_{i}^{2}+\varepsilon \beta\right)\left(\tilde{w}_{i-2}-8 \tilde{w}_{i-1}+8 \tilde{w}_{i+1}-\tilde{w}_{i+2}\right) \tilde{w}_{i} \\
= & \sum_{i=1}^{N-2}\left(\xi_{i+2}^{2}+\varepsilon \beta\right) \tilde{w}_{i} \tilde{w}_{i+2}-8 \sum_{i=1}^{N-1}\left(\xi_{i+1}^{2}+\varepsilon \beta\right) \tilde{w}_{i} \tilde{w}_{i+1} \\
& +8 \sum_{i=1}^{N-1}\left(\xi_{i}^{2}+\varepsilon \beta\right) \tilde{w}_{i+1} \tilde{w}_{i}-\sum_{i=1}^{N-2}\left(\xi_{i}^{2}+\varepsilon \beta\right) \tilde{w}_{i+2} \tilde{w}_{i} \\
= & \sum_{i=1}^{N-2}\left(2 \xi_{i}+2 \Delta \xi\right) 2 \Delta \xi \tilde{w}_{i+2} \tilde{w}_{i}-8 \sum_{i=1}^{N-1}\left(2 \xi_{i}+\Delta \xi\right) \Delta \xi \tilde{w}_{i+1} \tilde{w}_{i} .
\end{aligned}
$$

As a consequence, we get

$$
L\left(\tilde{w}^{\Delta}\right) \cdot \tilde{w}^{\Delta}=\sum_{i=1}^{N-2} a_{i}\left(-\tilde{w}_{i} \tilde{w}_{i+2}\right)+\sum_{i=1}^{N-1} b_{i} \tilde{w}_{i} \tilde{w}_{i+1}-\frac{30 \varepsilon \beta}{12 \Delta \xi^{2}} \sum_{i=1}^{N} \tilde{w}_{i}^{2},
$$

where we have set

$$
a_{i}=\frac{1}{3}\left(\frac{\varepsilon \beta}{2 \Delta \xi^{2}}-1\right)\left(\xi_{i}+\Delta \xi\right) \text { and } b_{i}=\frac{4}{3}\left(\frac{2 \varepsilon \beta}{\Delta \xi^{2}}-1\right)\left(\xi_{i}+\frac{\Delta \xi}{2}\right)
$$

Using (2.25), we have

$$
\begin{aligned}
\sum_{i=1}^{N-2} a_{i}\left(-\tilde{w}_{i} \tilde{w}_{i+2}\right)= & \sum_{i=-1}^{N} a_{i}\left(-\tilde{w}_{i} \tilde{w}_{i+2}\right) \\
= & -\frac{1}{2} \sum_{i=-1}^{N} a_{i}\left(\tilde{w}_{i+2}-2 \tilde{w}_{i+1}+\tilde{w}_{i}\right)^{2}+\frac{1}{2} \sum_{i=0}^{N}\left(a_{i}+a_{i-1}\right)\left(\tilde{w}_{i+1}-\tilde{w}_{i}\right)^{2} \\
& -\sum_{i=0}^{N}\left(a_{i}+a_{i-1}\right) \tilde{w}_{i} \tilde{w}_{i+1}+\sum_{i=1}^{N} a_{i-1} \tilde{w}_{i}^{2}
\end{aligned}
$$


to obtain

$$
\begin{array}{r}
L\left(\tilde{w}^{\Delta}\right) \cdot \tilde{w}^{\Delta}=-\frac{1}{2} \sum_{i=-1}^{N} a_{i}\left(\tilde{w}_{i+2}-2 \tilde{w}_{i+1}+\tilde{w}_{i}\right)^{2}+\frac{1}{2} \sum_{i=0}^{N}\left(a_{i}+a_{i-1}\right)\left(\tilde{w}_{i+1}-\tilde{w}_{i}\right)^{2} \\
+\sum_{i=0}^{N}\left(b_{i}-a_{i}-a_{i-1}\right) \tilde{w}_{i} \tilde{w}_{i+1}+\sum_{i=1}^{N}\left(a_{i-1}-\frac{30 \varepsilon \beta}{12 \Delta \xi^{2}} \xi_{i}\right) \tilde{w}_{i}^{2} .
\end{array}
$$

Moreover, from a straightforward computation, we get

$$
\begin{aligned}
\sum_{i=0}^{N}\left(b_{i}-a_{i}-a_{i-1}\right) \tilde{w}_{i} \tilde{w}_{i+1}=\frac{1}{2} \sum_{i=1}^{N}( & \left.b_{i}+b_{i-1}-a_{i}-2 a_{i-1}-a_{i-2}\right) \tilde{w}_{i}^{2} \\
& -\frac{1}{2} \sum_{i=0}^{N}\left(b_{i}+-a_{i}+a_{i-1}\right)\left(\tilde{w}_{i+1}-\tilde{w}_{i}\right)^{2},
\end{aligned}
$$

to write

$$
\begin{gathered}
L\left(\tilde{w}^{\Delta}\right) \cdot \tilde{w}^{\Delta}=-\frac{1}{2} \sum_{i=-1}^{N} a_{i}\left(\tilde{w}_{i+2}-2 \tilde{w}_{i+1}+\tilde{w}_{i}\right)^{2}-\frac{1}{2} \sum_{i=0}^{N}\left(b_{i}-2 a_{i}+2 a_{i-1}\right)\left(\tilde{w}_{i+1}-\tilde{w}_{i}\right)^{2} \\
+\sum_{i=1}^{N}\left(\frac{1}{2}\left(b_{i}-a_{i}+b_{i-1}-a_{i-2}\right)-\frac{30 \varepsilon \beta}{12 \Delta \xi^{2}} \xi_{i}\right) \tilde{w}_{i}^{2} .
\end{gathered}
$$

Arguing (3.17), we obtain the required equality (3.14). The proof is thus completed. 口

LEMMA 6. Let $L$ be the linear map defined by (3.10). Then, there exists a constant $\Lambda>0$, which depends on $\Delta \xi, \xi^{\star}, \ell, w^{\star}$ and $w_{R}$, such that

$$
L\left(r^{\Delta}\right) \cdot \tilde{w}^{\Delta} \leq \Lambda \sqrt{\frac{\ell-\xi^{\star}}{\Delta \xi}}\left\|\tilde{w}^{\Delta}\right\| .
$$

We omit here the proof of the above result since it turns out to follow the same guidelines as the one of Lemma 3.

Equipped with the two above technical lemmas, we now give the proof of Theorem 2.

Proof. First, since the function $p$ is smooth enough, let us underline that Lemma 4 applies. As a consequence, the estimation (2.29) with (2.30) holds but for $M_{p^{\prime}}$ instead of $M_{f^{\prime}}$.

Now, gathering estimates (3.14), (3.18) and (2.29) with $p$ in place of $f$, provides

$$
\begin{aligned}
\tilde{\mathcal{E}}\left(\tilde{w}^{\Delta}\right) \cdot \tilde{w}^{\Delta} \leq & -\frac{1}{6}\left(\frac{\varepsilon \beta}{2 \Delta \xi^{2}}-1\right) \sum_{i=-1}^{N} \xi_{i+1}\left(\tilde{w}_{i+2}-2 \tilde{w}_{i+1}+\tilde{w}_{i}\right)^{2} \\
& -\frac{\varepsilon \beta}{\Delta \xi^{2}} \sum_{i=0}^{N}\left(\xi_{i}+\frac{\Delta \xi}{2}\right)\left(\tilde{w}_{i+1}-\tilde{w}_{i}\right)^{2}-\sum_{i=1}^{N} \xi_{i} \tilde{w}_{i}^{2} \\
& +\frac{97 M_{p^{\prime}}}{24 \Delta \xi} \sum_{i=0}^{N}\left(\tilde{w}_{i+1}-\tilde{w}_{i}\right)^{2}+\sqrt{\frac{\ell-\xi^{\star}}{\Delta \xi}}\left\|\tilde{w}^{\Delta}\right\|+A_{0} .
\end{aligned}
$$


Arguing the discrete Poincaré estimations (2.34) and (2.36), as soon as $\Delta \xi \leq \varepsilon \beta$, we get

$$
\tilde{\mathcal{E}}\left(\tilde{w}^{\Delta}\right) \cdot \tilde{w}^{\Delta} \leq-\left(\xi^{\star}+\alpha \frac{\Delta \xi^{2}}{\left(\ell-\xi^{\star}\right)^{2}}\right)\left\|\tilde{w}^{\Delta}\right\|^{2}+\sqrt{\frac{\ell-\xi^{\star}}{\Delta \xi}}\left\|\tilde{w}^{\Delta}\right\|+A_{0},
$$

with

$$
\alpha=\frac{\xi^{\star}}{6}\left(\frac{\varepsilon \beta}{2 \Delta \xi^{2}}-1\right) \frac{\Delta \xi^{2}}{4\left(\ell-\xi^{\star}\right)^{2}}+\frac{\varepsilon \beta}{\Delta \xi^{2}}\left(\xi^{\star}+\frac{\Delta \xi}{2}\right)-\frac{97 M_{f^{\prime}}}{24 \Delta \xi} .
$$

Since $\xi^{\star}$ is positive, the right-hand side in the above estimation turns out to be a quadratic function with respect to $\left\|\tilde{w}^{\Delta}\right\|$, with a negative leading order term. As a consequence, there exists $k>0$, large enough, such that $\tilde{\mathcal{E}}\left(\tilde{w}^{\Delta}\right) \cdot \tilde{w}^{\Delta} \leq 0$ for all $\tilde{w}^{\Delta} \in \mathbb{R}^{N}$ with $\left\|\tilde{w}^{\Delta}\right\|=k$. Arguing Lemma 1, there exists $\tilde{w}^{\Delta} \in \mathbb{R}^{N}$ solution of $\tilde{\mathcal{E}}\left(\tilde{w}^{\Delta}\right)=0$. The proof is thus achieved.

4. Numerical experiments. In this section, we present numerical results in both scalar and system cases.

First, we detail the adopted continuation-type Newton method. For large values of $\varepsilon$, in practice $\varepsilon=\mathcal{O}(1)$, the nonlinear scheme (2.7), respectively (3.6), turns out to be easily solvable arguing a Newton method. Next, the obtained solution with a large value of $\varepsilon$ is considered to initialize the Newton algorithm for a smaller value of $\varepsilon$. We iterate such a process, by decreasing $\varepsilon$ until the expected value of $\varepsilon$ is reached. Moreover, in order to get better convergence, we have adopted here a damping Newton method given in [19] (see also $[4,18]$ ).

In the following subsections, we use this method to solve Riemann problems in the scalar and then in the system framework for several flux functions. In order to be consistent with a bounded derivative of the flux function, as assumed in Theorems 1 and 2 , the function $f$ (respectively $p$ ) is defined as follows:

$$
f(w)= \begin{cases}f^{0}(w) & \text { if } w \in\left[w_{\min }, w_{\max }\right], \\ f^{\operatorname{ext}}(w) & \text { otherwise. }\end{cases}
$$

The function $f^{0}$ is given, while $f^{\text {ext }}$ is a continuous extension in order to enforce the derivative to be bounded. In addition, $w_{\min }$ and $w_{\max }$ are chosen large enough such that the performed simulations are entirely contained within the interval $\left[w_{\min }, w_{\max }\right]$. As a consequence, the continuous extension of $f^{0}$ is never considered.

4.1. Scalar case. The first experiment consists in applying our method to problems studied in [8] by considering (2.1) with $f^{0}(w)=w^{3}$. In Figure 4.1, we present the results obtained for a classical solution with the following parameters: $w_{L}=4$, $w_{R}=2, N=1000$ and $\beta=1$. The simulation is performed over the domain $\mathcal{D}=[10,40]$. In this classical test case, $\gamma$ has no influence on the solution. We verify this property by considering two values of $\gamma: 1$ and 10 . These results are obtained with $\varepsilon=10^{-3}$, and compared to the exact solution. As expected, the numerical scheme converges to the right solution, the shock being at $\xi=28=\frac{f\left(w_{L}\right)-f\left(w_{R}\right)}{w_{L}-w_{R}}$.

We are now interested in non classical solutions and consider the Riemann problem given by $w_{L}=4, w_{R}=-2$. In Figure 4.2 top left, we present solutions obtained for different values of $\varepsilon: 5,1$ and $10^{-3}$. Parameters are $\mathcal{D}=[0,60], N=2000, \beta=1$ and $\gamma=1$. For $\varepsilon=5$, the obtained solution is smooth (and the Newton method converges 


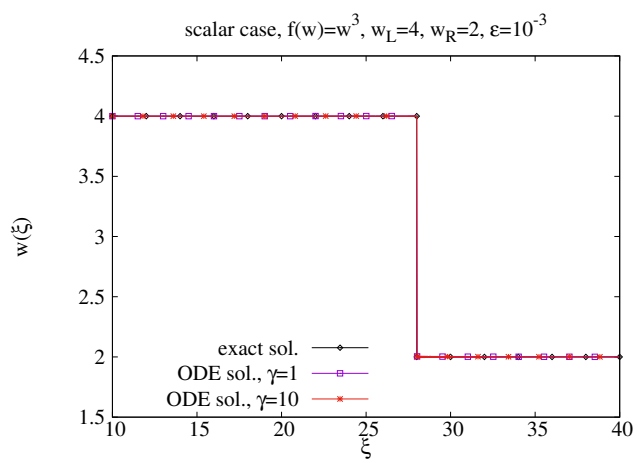

FIG. 4.1. Classical solution in a scalar case $f^{0}(w)=w^{3}$.

easily). Then, when $\varepsilon$ diminishes, the solution becomes rougher and converges to the exact one, constituted of two shocks. Note that the exact value of the intermediate state, that we denote by $w^{\star}$, is given by $w^{\star}=-w_{L}+\frac{\sqrt{2}}{3} \approx-3.5286$ as explained in [8]. Then, the solution is constituted of two shocks, one at $\xi=\frac{f\left(w_{L}\right)-f\left(w^{\star}\right)}{w_{L}-w^{\star}} \approx 14.3366$, the other at $\xi=\frac{f\left(w_{R}\right)-f\left(w^{\star}\right)}{w_{R}-w^{\star}} \approx 23.5082$.

In addition, we verify that as soon as the length of the interval is large enough to contain all the waves involved in the Riemann problem, the numerical approximation does not depend on the value of $\ell$. We illustrate this purpose in Figure 4.2 top right.

In Figure 4.2 bottom, we present a non classical solution constituted of a shock and a rarefaction wave, obtained for the Riemann problem given by $w_{L}=2, w_{R}=-2$. Parameters are $\mathcal{D}=[0,60], N=2000, \beta=1$ and the solutions are plotted for $\varepsilon=10^{-3}$. We look at the influence of $\gamma$ which is taken equal to $0.5,1$ and 5 . As for the previous test cases, solutions are very similar to the exact ones. Note that the exact solution presents an intermediate state that depends on $\gamma$ and that is given by $w^{\star}=-w_{L}+\frac{\sqrt{2}}{3 \sqrt{\gamma}}$. Then, the solution presents a shock on the left of the intermediate state and a rarefaction wave on the right. The velocity of the shock is $w_{L}^{2}+w_{L} w^{\star}+w^{\star 2}$. Concerning the rarefaction wave, it is given by

$$
\begin{cases}w^{\star}, & \xi \leq f^{\prime}\left(w^{\star}\right) \\ f^{\prime-1}(\xi), & f^{\prime}\left(w^{\star}\right) \leq \xi \leq f^{\prime}\left(w_{R}\right) \\ w_{R}, & f^{\prime}\left(w_{R}\right) \leq \xi\end{cases}
$$

In our cases, we have $f^{0^{\prime}}(w)=3 w^{2}$ and $\left(f^{0^{\prime}}\right)^{-1}(\xi)=-\sqrt{\frac{\xi}{3}}$, which determines the exact solution [8], for each value of $\gamma$.

Next, in order to illustrate the behavior of the numerical solution, in the limit of $\Delta \xi$ to zero, we present some numerical results. Indeed, because of the capillarity term, this is a very difficult task. Two distinct situations must be considered. Firstly, consider a solution where the dispersive term does not dominate the solution. For such a situation, no oscillations are expected. For instance, let us consider a scalar rarefaction wave with left and right states given by $w_{L}=2, w_{R}=4$, where we have set $\gamma=\beta=1$. The solution is presented in Figure 4.3 for two distinct values of $\varepsilon$.

In Tables 4.1 and 4.2 , the errors with respect to $\Delta \xi$ coming with these numerical tests are given. As expected, we notice that $L^{1}$ and $L^{2}$ errors saturate proportionally to the value of $\varepsilon$. Indeed, these errors are evaluated by comparing the approximated solution with non vanishing $\varepsilon$ to the exact solution obtained in the limit of $\varepsilon=0$. In 


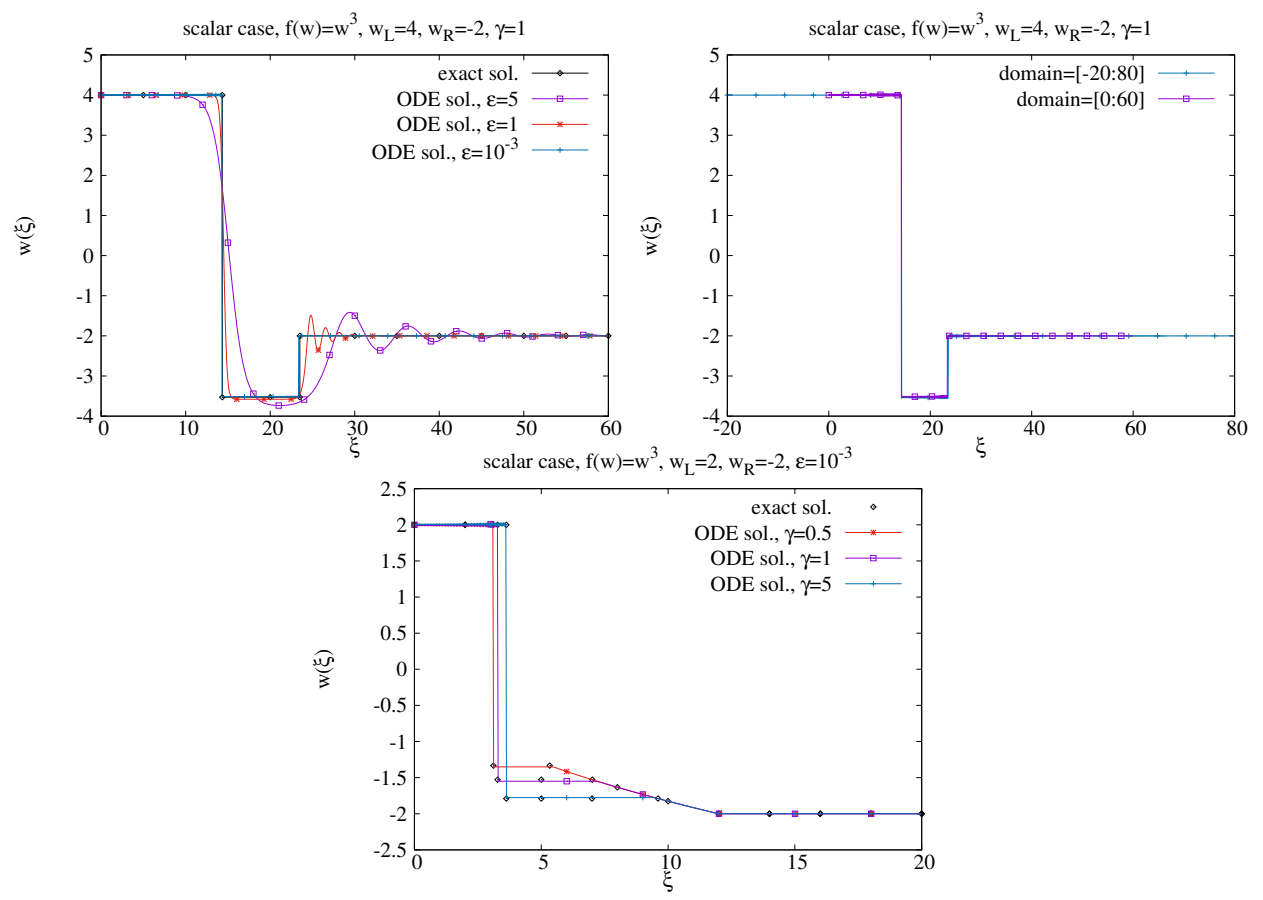

FIG. 4.2. Non classical solutions in scalar cases where $f^{0}(w)=w^{3}$.
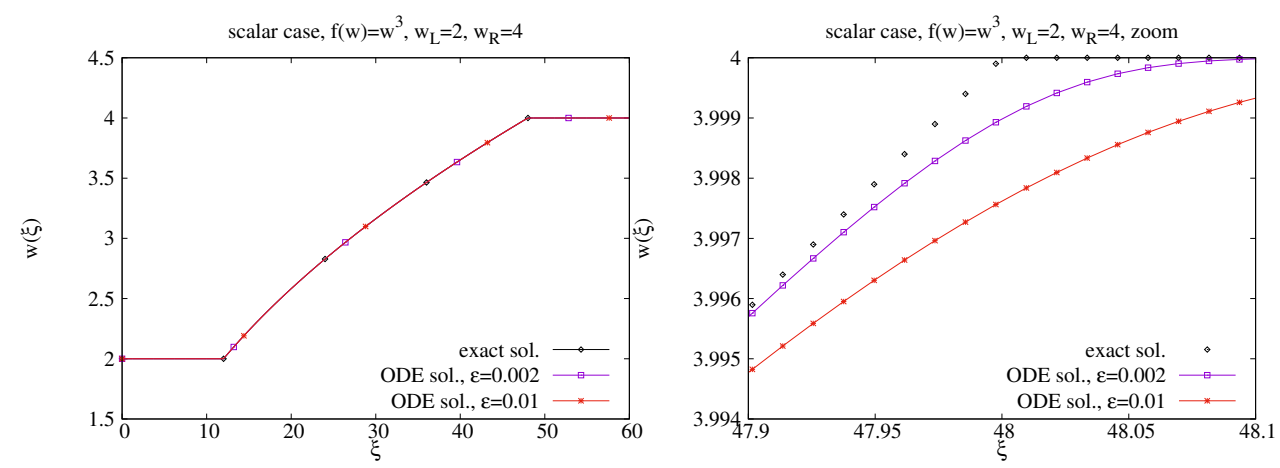

FIG. 4.3. Classical rarefaction wave in scalar cases where $f^{0}(w)=w^{3}$.

addition, the convergence seems to be reached very quickly because of the 4-order of the scheme. We remark the strange behavior of the $L^{\infty}$ error with a small increasing. We suppose that this behavior comes from the lack of smoothness of the exact solution which is $C^{0}$ but not $C^{1}$. It seems that the $L^{\infty}$ error is not relevant for this numerical experiment.

Secondly, let us consider a solution where the dissipative term is dominant. From the work by Joseph and LeFloch [11], the solution converges pointwise as $\varepsilon \rightarrow 0$ to a weak solution of the associated hyperbolic conservation law. As a consequence, the associated behavior of the solution is expected to tend to 0 with an infinite frequency of oscillations. However, the measure of the convex hull of the oscillations may not converge to 0 . Now, from a numerical point of view, up to our knowledge, there is no suitable norm to evaluate the behavior of the solution as $\varepsilon \rightarrow 0$. Indeed, because of 


\begin{tabular}{|l|l|l|l|}
\hline$\Delta \xi$ & $L^{\infty}$ error & $L^{1}$ error & $L^{2}$ error \\
\hline $6.0060 \mathrm{E}-002$ & $1.5185 \mathrm{E}-003$ & $3.3016 \mathrm{E}-004$ & $4.5806 \mathrm{E}-004$ \\
$4.0026 \mathrm{E}-002$ & $1.7250 \mathrm{E}-003$ & $3.2972 \mathrm{E}-004$ & $4.7346 \mathrm{E}-004$ \\
$3.0015 \mathrm{E}-002$ & $1.8059 \mathrm{E}-003$ & $3.2952 \mathrm{E}-004$ & $4.7532 \mathrm{E}-004$ \\
$2.0006 \mathrm{E}-002$ & $1.8813 \mathrm{E}-003$ & $3.2934 \mathrm{E}-004$ & $4.7563 \mathrm{E}-004$ \\
$1.5003 \mathrm{E}-002$ & $1.9180 \mathrm{E}-003$ & $3.2927 \mathrm{E}-004$ & $4.7553 \mathrm{E}-004$ \\
$1.2002 \mathrm{E}-002$ & $1.9404 \mathrm{E}-003$ & $3.2924 \mathrm{E}-004$ & $4.7545 \mathrm{E}-004$ \\
\hline \multicolumn{4}{|c}{ TABLE 4.1} \\
Errors for $\varepsilon=0.002$.
\end{tabular}

\begin{tabular}{|l|l|l|l|}
\hline$\Delta \xi$ & $L^{\infty}$ error & $L^{1}$ error & $L^{2}$ error \\
\hline $6.0060 \mathrm{E}-002$ & $4.3057 \mathrm{E}-003$ & $1.7892 \mathrm{E}-003$ & $1.7052 \mathrm{E}-003$ \\
$4.0026 \mathrm{E}-002$ & $4.4570 \mathrm{E}-003$ & $1.7886 \mathrm{E}-003$ & $1.7053 \mathrm{E}-003$ \\
$3.0015 \mathrm{E}-002$ & $4.5321 \mathrm{E}-003$ & $1.7883 \mathrm{E}-003$ & $1.7049 \mathrm{E}-003$ \\
$2.0006 \mathrm{E}-002$ & $4.6074 \mathrm{E}-003$ & $1.7881 \mathrm{E}-003$ & $1.7045 \mathrm{E}-003$ \\
$1.5003 \mathrm{E}-002$ & $4.6454 \mathrm{E}-003$ & $1.7880 \mathrm{E}-003$ & $1.7043 \mathrm{E}-003$ \\
$1.2002 \mathrm{E}-002$ & $4.6682 \mathrm{E}-003$ & $1.7880 \mathrm{E}-003$ & $1.7043 \mathrm{E}-003$ \\
\hline \multicolumn{4}{|c|}{ TABLE 4.2} \\
\hline
\end{tabular}

the comparison with discontinuous limit solutions, the $L^{\infty}$ norm is not relevant. Next, because of the convex hull of the oscillations, both $L^{1}$ and $L^{2}$ norms are once again not relevant. To illustrate our purpose, we adopt the approximation of a solution containing a non classical shock discontinuity where $w_{L}=4$ and $w_{R}=-2$, with $\beta=\gamma=1, \varepsilon=0.01$ and $N=2000$ or $N=4000$. Around the shock, the dissipative term introduces oscillations as presented in Figure 4.4.

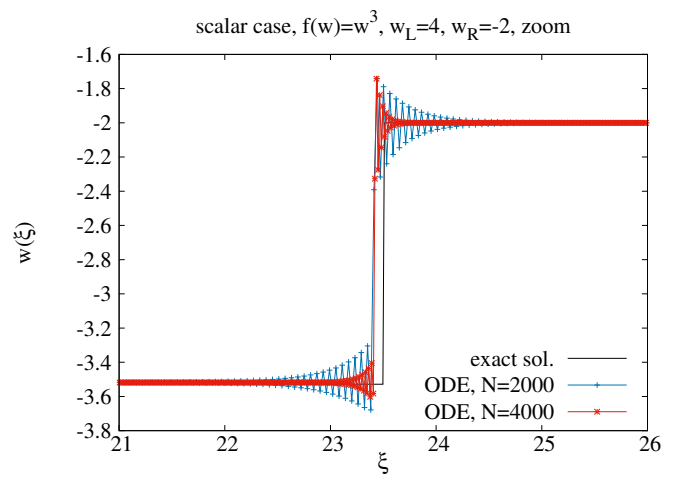

FIG. 4.4. Zoom on a non classical shock wave in scalar cases where $f^{0}(w)=w^{3}$.

We can notice that even with fine mesh, as soon as $\varepsilon$ is small enough, the dispersive oscillations are not accurately captured because of a very high frequency. In the above example, each oscillation is approximated with only one point, which is clearly insufficient (for instance, see the paper of Ernest, LeFloch and Mishra [8], Figures $2.4,2.5$ or 2.7$)$. As a consequence, at this level, it seems that a $\Delta \xi$ convergence is not reachable, except by considering extremely fine meshes which is not the purpose of this work.

The second series of experiments consists in the study of $(2.1)$ with $f^{0}(w)=w^{3}-w$ 
on the domain $\mathcal{D}$, as in [5]. We first consider the Riemann problem given by $w_{L}=1$, $w_{R}=-5$ on $\mathcal{D}=[-60,120]$. The solution obtained at $\varepsilon=10^{-2}$, with $N=2000$, $\beta=5$ and $\gamma=37.5$ (as in [5] Figure 3.6) is plotted on Figure 4.5 left. This solution is compared to the one of a Finite Volumes scheme with a Lax-Friedrichs flux with the following parameters: $N=5 \times 10^{4}$ and a time step $\Delta t=2 \times 10^{-5}$. Then we consider the Riemann problem given by $w_{L}=0, w_{R}=2$ on $\mathcal{D}=[-60,60]$. On Figure 4.5 right, we present the solution given by our method and the one given by a Lax-Friedrichs Finite Volumes scheme. The parameters are unchanged.
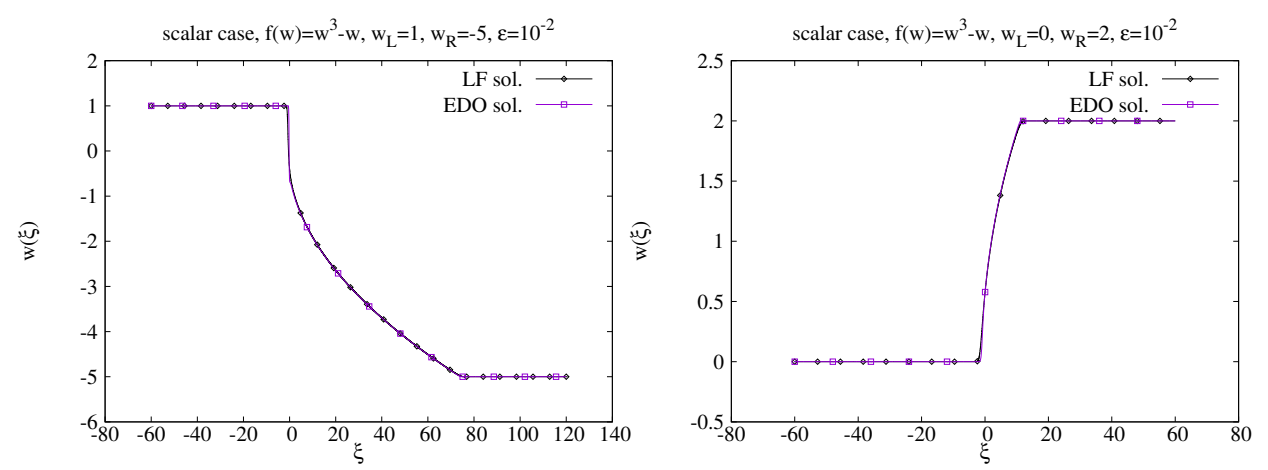

FIG. 4.5. Classical solutions and comparison with a Lax-Friedrichs Finite Volumes scheme in the case $f^{0}(w)=w^{3}-w$.

Now, we reproduce Figures 3.6 and 3.7 of [5] by considering different values of $w_{L}$ and of $\beta$. On Figure 4.6 left, we consider different Riemann problems with $w_{R}=-5$ and $w_{L}$ varying from 1 to 4 , on $\mathcal{D}=[-60,120]$. Other parameters are fixed to $\varepsilon=10^{-2}, N=2000, \beta=5$ and $\gamma=37.5$. As explained in [5], the solution is classical when $w_{L}=1$ but becomes non classical when $w_{L}=2$ or more. The influence of $\beta$ is shown on Figure 4.6 right: $w_{L}$ is fixed to 4 but $\beta$ varies from 5 to 30. By increasing $\beta$, we go from a non classical solution to a classical one, as presented in [5].
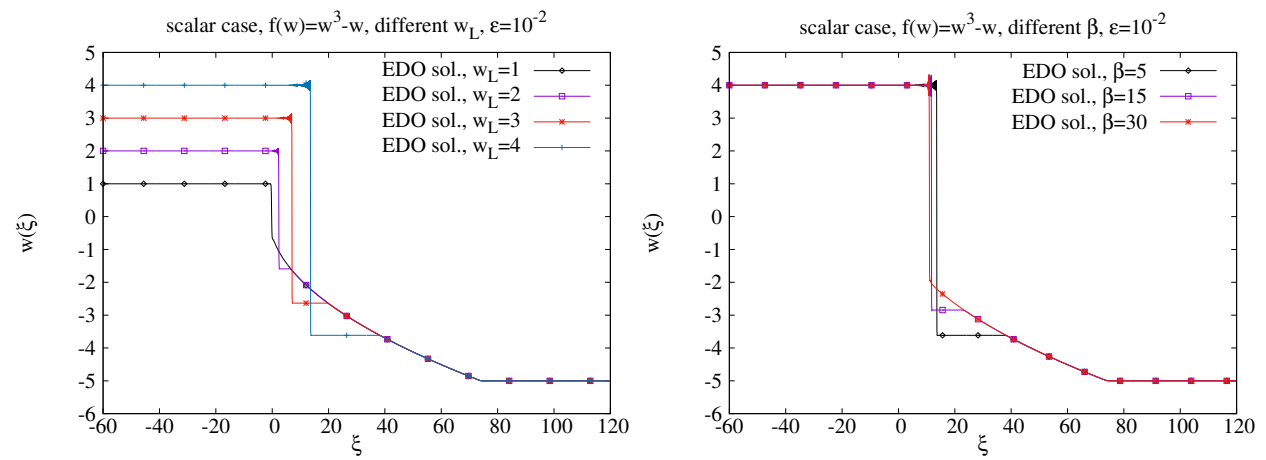

FIG. 4.6. From classical to non classical solutions in the case $f^{0}(w)=w^{3}-w$.

The third scalar framework we are interested in is the Burgers equation, by considering $f^{0}(w)=\frac{w^{2}}{2}$. This problem only admits classical solutions. On Figure 4.7 left, we represent the stationary shock solution of the Riemann problem given by $w_{L}=2$, $w_{R}=-2$ on $\mathcal{D}=[-10,10]$. The one given by our method for $\gamma=1$ and $\gamma=10$ is compared to the one of a Lax-Friedrichs Finite Volumes scheme. Parameters are the following: $\varepsilon=10^{-3}, N=2000$ and $\beta=1$ for our method; $N=10^{4}$ and a time step 
$\Delta t=10^{-4}$ for the Finite Volumes scheme. Even if oscillations appear, the shock is well reproduced. A non stationary shock solution of the Riemann problem given by $w_{L}=5$ and $w_{R}=1$ is represented on Figure 4.7 right. Parameters are unchanged. As previously, the shock is well represented except that some oscillations appear.
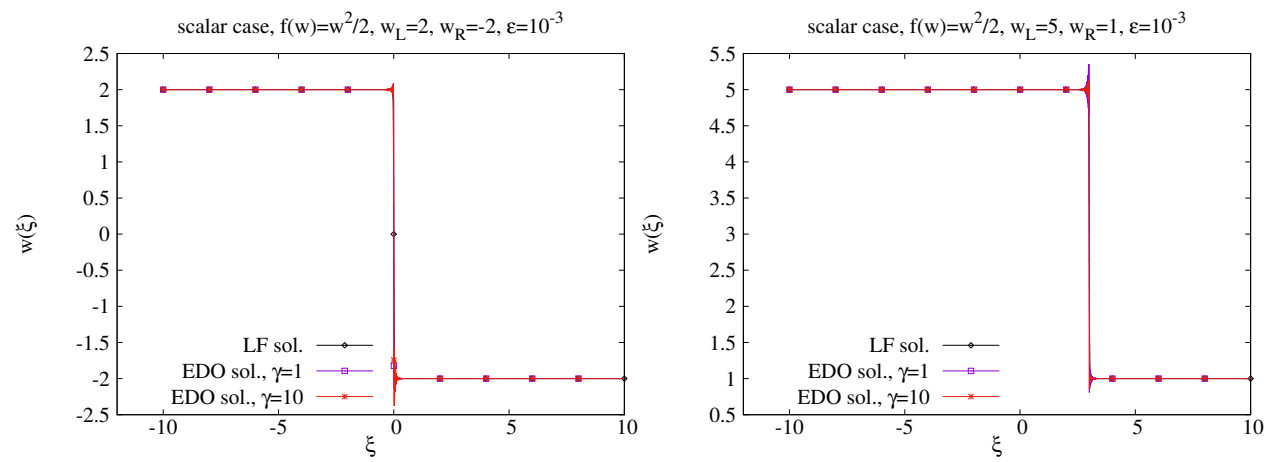

Fig. 4.7. Classical stationary and non stationary shocks in the case $f^{0}(w)=\frac{w^{2}}{2}$.

Finally, we study a rarefaction case by considering the Riemann problem given by $w_{L}=-2, w_{R}=2$. Solutions are plotted on Figure 4.8 for the same parameters as previously. The rarefaction wave is well captured by our method and does not suffer from diffusion, contrary to the one given by the Lax-Friedrichs Finite Volumes scheme.

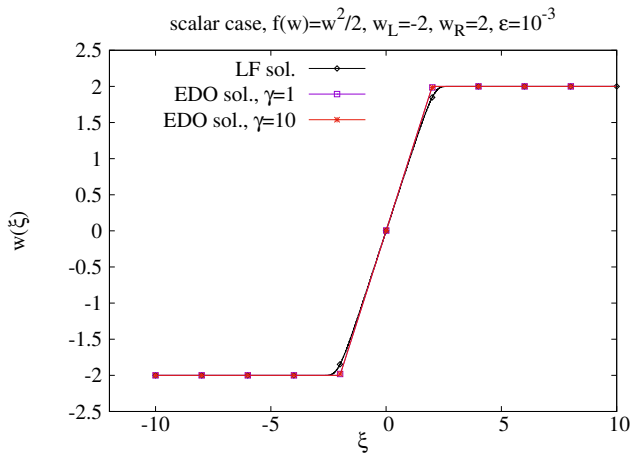

FiG. 4.8. Classical rarefaction wave in the case $f^{0}(w)=\frac{w^{2}}{2}$.

4.2. System case. In this subsection, we are interested in the behavior of our method in the p-system model. More precisely, we use the method presented in Section 3 to solve equation (3.3) in the case $p^{0}(w)=\frac{w^{3}}{3}+w$ on $\mathcal{D}=[-\ell, \ell]$. As explained in Section 3 and in [11], we cannot consider $\xi=0$, so that we discretize (3.3) over the two intervals $\left[-\ell,-\xi^{\star}\right]$ and $\left[\xi^{\star}, \ell\right]$. First, we consider the Riemann problem given by $w_{L}=1, w_{R}=5$ and $\ell=8$. On Figure 4.9 left, we present the solution obtained by our method with two values of $\xi^{\star}: 0.8$ and 1.6, and compare it to the solution given by a Lax-Friedrichs Finite Volumes scheme. Parameters are the following: $\varepsilon=4 \times 10^{-2}$, $N=1000, \beta=1, \gamma=1$ for our method; $N=10^{4}$ and a time step $\Delta t=10^{-4}$ for the Finite Volumes solutions. For this set of parameters, the solution is classical so that it is well represented by refined enough Lax-Friedrichs scheme. This let us verify that for small enough $\xi^{\star}$, our method gives very good results on this kind of 
p-system problem. Moreover, the solution does not change with $\xi^{\star}$, if this one is small enough. Finally, we are interested in non classical solutions. We consider the following parameters: $w_{L}=-3, w_{R}=10, \ell=20, \beta=1, \gamma=20, \varepsilon=2.5 \times 10^{-2}$ and $N=1000$. Parameters for the Lax-Friedrichs scheme are $N=10^{4}$ and $\Delta t=10^{-4}$. The solutions given by both methods are plotted on Figure 4.9 right. As we can see, the Lax-Friedrichs scheme is not able to capture the non classical solution, whereas our method approaches the solution with three distinct waves.
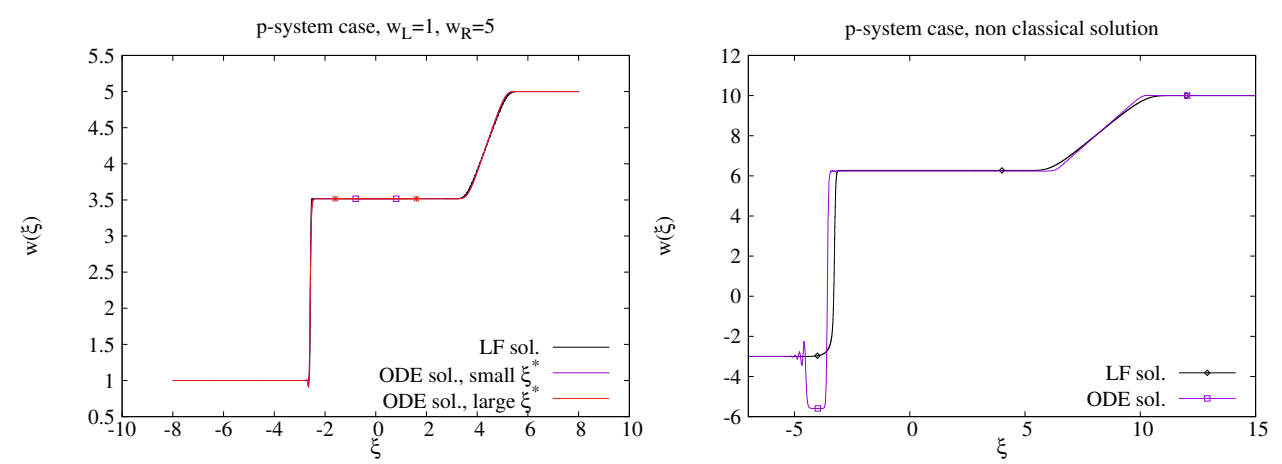

FIG. 4.9. Solutions in the p-system case with $p^{0}(w)=\frac{w^{3}}{3}+w$. In the left figure, the magenta curve corresponds to $\xi^{\star}=0.8$ displayed by the magenta squares, whereas the red curve corresponds to $\xi^{\star}=1.6$ displayed by the red crosses. The right figure presents a non classical solution.

Acknowledgement. Authors would like to thanks Guy Moebs for his helpful contribution to the code optimization. Otherwise, they thank the Centre Henri Lebesgue ANR-11-LABX-0020-01 for its stimulating mathematical research programs and M. Bessemoulin-Chatard and A. Crestetto are supported by the ANR (The French National Research Agency) Project MoHyCon ANR-17-CE40-0027-01. In addition, we would like to thank the anonymous referee for very fruitful comments and discussions.

\section{REFERENCES}

[1] W.-J. Beyn. The numerical computation of connecting orbits in dynamical systems. IMA Journal of Numerical Analysis, 10(3):379-405, 1990.

[2] B. Boutin, C. Chalons, F. Lagoutière, and P. G. LeFloch. Convergent and conservative schemes for nonclassical solutions based on kinetic relations. Interfaces and free boundaries, 10(3):399-421, 2008.

[3] B. Boutin, F. Coquel, and P. G. LeFloch. Coupling techniques for nonlinear hyperbolic equations. I self-similar diffusion for thin interfaces. Proceedings of the Royal Society of Edinburgh: Section A Mathematics, 141(05):921-956, 2011.

[4] Oleg Burdakov. Some globally convergent modifications of Newton's method for solving systems of nonlinear equations. In Soviet mathematics-Doklady, volume 22, pages 376-378, 1980.

[5] C. Chalons and P. G. LeFloch. A fully discrete scheme for diffusive-dispersive conservation laws. Numerische Mathematik, 89(3):493-509, 2001.

[6] C. M. Dafermos. Hyperbolic conservation laws in continuum physics, volume 325 of Grundlehren der Mathematischen Wissenschaften [Fundamental Principles of Mathematical Sciences]. Springer-Verlag, Berlin, third edition, 2010.

[7] R. J. DiPerna. Uniqueness of solutions to hyperbolic conservation laws. Indiana Univ. Math. J., 28(1):137-188, 1979.

[8] J. Ernest, P. LeFloch, and S. Mishra. Schemes with well controlled dissipation (wcd) I: Nonclassical shock waves. In Seminar fr Angewadte Mathematil, 2013.

[9] L. C. Evans. Partial differential equations, volume 19 of Graduate Studies in Mathematics. American Mathematical Society, Providence, RI, 1998.

[10] E. Godlewski and P.-A. Raviart. Numerical approximation of hyperbolic systems of conser- 
vation laws, volume 118 of Applied Mathematical Sciences. Springer-Verlag, New York, 1996.

[11] K. T Joseph and P. G. LeFloch. Singular limits in phase dynamics with physical viscosity and capillarity. In Proceedings of the Royal Society of Edinburgh-A-Mathematics, volume 137, pages 1287-1312. Royal Society of Edinburgh, 2007.

[12] P.D. Lax. Shock waves and entropy. In Contributions to nonlinear functional analysis (Proc. Sympos., Math. Res. Center, Univ. Wisconsin, Madison, Wis., 1971), pages 603-634. Academic Press, New York, 1971.

[13] Peter D Lax. Hyperbolic systems of conservation laws and the mathematical theory of shock waves, volume 11. SIAM, 1973.

[14] P. G. LeFloch. Hyperbolic systems of conservation laws. Lectures in Mathematics ETH Zürich. Birkhäuser Verlag, Basel, 2002. The theory of classical and nonclassical shock waves.

[15] P. G. LeFloch and S. Mishra. Numerical methods with controlled dissipation for small-scale dependent shocks. Acta Numerica, 23:743-816, 2014.

[16] P. G. LeFloch and C. Rohde. High-order schemes, entropy inequalities, and nonclassical shocks. SIAM Journal on Numerical Analysis, 37(6):2023-2060, 2000.

[17] P. G. LeFloch and C. Rohde. Zero diffusion-dispersion limits for self-similar Riemann solutions to hyperbolic systems of conservation laws. Indiana University mathematics journal, 50(4):1707-1743, 2001.

[18] James M Ortega and Werner C Rheinboldt. Iterative solution of nonlinear equations in several variables, volume 30. Siam, 1970.

[19] Daniel Ralph. Global convergence of damped Newton's method for nonsmooth equations via the path search. Mathematics of Operations Research, 19(2):352-389, 1994.

[20] S. Schecter, B. J. Plohr, and D. Marchesin. Computation of Riemann solutions using the Dafermos regularization and continuation. Discrete and Continuous Dynamical Systems, 10:965-986, 2004.

[21] S. Schecter and M. R. Taylor. Dafermos regularization of a diffusive-dispersive equation with cubic flux. Discrete and Continuous Dynamical Systems, 32(12):4069-4110, 2012. 